\title{
Massive identification of asteroids in three-body resonances
}

\author{
E. A. Smirnov and I. I. Shevchenko* \\ Pulkovo Observatory of the Russian Academy of Sciences \\ Pulkovskoje ave. 65, St. Petersburg 196140, Russia
}

\begin{abstract}
An essential role in the asteroidal dynamics is played by the mean motion resonances. Two-body planet-asteroid resonances are widely known, due to the Kirkwood gaps. Besides, so-called three-body mean motion resonances exist, in which an asteroid and two planets participate. Identification of asteroids in three-body (namely, Jupiter-Saturn-asteroid) resonances was initially accomplished by D. Nesvorný and A. Morbidelli (1998), who, by means of visual analysis of the time behaviour of resonant arguments, found 255 asteroids to reside in such resonances. We develop specialized algorithms and software for massive automatic identification of asteroids in the three-body, as well as two-body, resonances of arbitrary order, by means of automatic analysis of the time behaviour of resonant arguments. In the computation of orbits, all essential perturbations are taken into account. We integrate the asteroidal orbits on the time interval of $100000 \mathrm{yr}$ and identify main-belt asteroids in the three-body Jupiter-Saturn-asteroid resonances up to the 6th order inclusive, and in the two-body Jupiter-asteroid resonances up to the 9th order inclusive, in the set of $\sim 250000$ objects from the "Asteroids - Dynamic Site" (AstDyS) database. The percentages of resonant objects, including extrapolations for higher-order resonances, are determined. In particular, the observed fraction of pure-resonant asteroids (those exhibiting resonant libration on the whole interval of integration) in the three-body resonances up to the 6 th order inclusive is $\approx 0.9 \%$ of the whole set; and, using a higher-order extrapolation, the actual total fraction of pure-resonant asteroids in the three-body resonances of all orders is estimated as $\approx 1.1 \%$ of the whole set.
\end{abstract}

Keywords: Asteroids; Asteroids, dynamics; Celestial mechanics; Resonances

\section{Introduction}

A substantial role of resonances in the dynamics of asteroids became evident with the discovery of resonant "gaps" in the asteroid belt by D. Kirkwood in

*E-mail: iis@gao.spb.ru 
1867. The deepest minima in the distribution of asteroids in the semimajor axes of their orbits correspond to the mean motion resonances $2 / 1,3 / 1,4 / 1,5 / 2$, and $7 / 3$ with Jupiter. Mean motion resonance represents a commensurability between the mean frequencies of the orbital motions of an asteroid and a planet. Apart from the mean motion resonances, so-called secular resonances (Murray and Dermott, 1999; Morbidelli, 2002), representing commensurabilities between the precession rates of the orbits of an asteroid and a planet, are important in forming the dynamical structure of the asteroid belt.

There are two important classes of the mean motion resonances: apart from the usual (two-body) mean motion resonances of an asteroid and a planet, an appreciable role in the asteroidal dynamics is played by so-called three-body mean motion resonances (Murray et al., 1998; Nesvorný and Morbidelli, 1998, 1999; Morbidelli, 2002). In the latter case, the resonance represents a commensurability between the mean frequencies of the orbital motions of an asteroid and two planets (e.g., Jupiter and Saturn):

$$
m_{\mathrm{J}} \dot{\lambda_{\mathrm{J}}}+m_{\mathrm{S}} \dot{\lambda_{\mathrm{S}}}+m \dot{\lambda} \approx 0
$$

where $\dot{\lambda_{\mathrm{J}}}, \dot{\lambda_{\mathrm{S}}}, \dot{\lambda}$ are the time derivatives of the mean longitudes of Jupiter, Saturn, and asteroid, respectively, and $m_{\mathrm{J}}, m_{\mathrm{S}}, m$ are integers.

In view of the "overdensity" of the three-body resonances in the phase space of the asteroidal motion, Nesvorný and Morbidelli (1998) asserted that "the three-body mean motion resonances seem to be the main actors structuring the dynamics in the main asteroid belt".

Chaotic behaviour, which is often present in the dynamics of celestial bodies, is usually due to interaction of resonances (as in any Hamiltonian system, see Chirikov 1979), but not always it is known which are the interacting resonances that give rise to chaos. It is especially difficult to identify three-body resonances. How to distinguish between resonant and non-resonant motions? To solve this problem, a "resonant argument" (synonymously "resonant phase" or "critical argument") is introduced. It is a linear combination of some angular variables of a system under consideration; in the planar asteroidal problem it is given by

$$
\sigma_{p_{\mathrm{J}}, p_{\mathrm{S}}, p}=m_{\mathrm{J}} \lambda_{\mathrm{J}}+m_{\mathrm{S}} \lambda_{\mathrm{S}}+m \lambda+p_{\mathrm{J}} \varpi_{\mathrm{J}}+p_{\mathrm{S}} \varpi_{\mathrm{S}}+p \varpi,
$$

where $\lambda_{\mathrm{J}}, \lambda_{\mathrm{S}}, \lambda, \varpi_{\mathrm{J}}, \varpi_{\mathrm{S}}, \varpi$ are the mean longitudes and longitudes of perihelia of Jupiter, Saturn, and an asteroid, respectively, and $m_{\mathrm{J}}, m_{\mathrm{S}}, m, p_{\mathrm{J}}, p_{\mathrm{S}}, p$ are integers satisfying the D'Alembert rule (Morbidelli, 2002):

$$
m_{\mathrm{J}}+m_{\mathrm{S}}+m+p_{\mathrm{J}}+p_{\mathrm{S}}+p=0 .
$$

If resonant argument (2) librates (similarly to librations of a pendulum), the system is in resonance; if it circulates, the system is out of resonance. The motion of the system at the border between librations and rotations corresponds to the separatrix. Thus the pendulum dynamics provides a graphical model of resonance. In a certain sense this model of resonance is "universal" (Chirikov, 1979). In particular, the motion in three-body resonances can be described in 
the perturbed pendulum model (Murray et al., 1998; Nesvorný and Morbidelli, 1998, 1999; Shevchenko, 2007).

An important parameter of a mean motion resonance is its order q, equal to the absolute value of the algebraic sum of the coefficients at the mean longitudes in the resonant argument:

$$
q=\left|m_{\mathrm{J}}+m_{\mathrm{S}}+m\right| .
$$

The resonant order $q$ is important, because it is the power in which the eccentricity is raised in the coefficient of the leading resonant term in the expansion of the perturbing function (Nesvorný and Morbidelli, 1998). The corresponding subresonance width (characterizing also its "strength") is proportional to the square root of this coefficient. Thus the value of $q$ determines this important property of the leading subresonance.

In the case of two-body resonances, the role of the resonant order $q$ (defined below in Section 6) is analogous: the coefficient of the leading resonant term is proportional to $e^{q}$ (Nesvorný and Morbidelli, 1998), where $e$ is the asteroidal eccentricity.

However note that, when there is no strong overlapping of subresonances, the resonant order $q$ is not related to the width of the whole resonant multiplet, because the separation of subresonances depends solely on the secular precession rates of the pericentres (Nesvorný and Morbidelli, 1999); thus the degree of overlap (and hence, chaos) in the multiplets is expected to asymptotically decrease with the resonant order (Nesvorný and Morbidelli, 1999; Morbidelli and Guzzo, 1997).

One may expect that, generally, broader the leading subresonance of a mean motion resonance, greater is the number of objects residing in this mean motion resonance. However, no strict correlation exists, due to a competition of various dynamical and physical processes, populating or depopulating the resonances. We shall discuss this further in more detail.

In our procedure of resonance identification, described in detail below, we limit the set of possible combinations of the integers $m_{\mathrm{J}}, m_{\mathrm{S}}, m$ by adopting the following conditions:

$$
\begin{gathered}
q \leq q_{\max }, \\
\left|m_{\mathrm{J}}\right|,\left|m_{\mathrm{S}}\right|,|m| \leq M_{\max },
\end{gathered}
$$

where $q_{\max }=6$ and $M_{\max }=8$.

Nesvorný and Morbidelli (1998) used condition (5) (presumably with $q_{\max }=$ 10, as follows from data in table 3 in Nesvorný and Morbidelli 1998). In (Nesvorný and Morbidelli, 1999), instead of (6), the following truncation condition was used:

$$
\left|m_{\mathrm{J}}\right|+\left|m_{\mathrm{S}}\right|+|m| \leq Q_{\max }
$$

(see eqs. (29) and (30) and comments on them in Nesvorný and Morbidelli 1999). 
We identify the three-body resonances in the current motion of asteroids with known orbital elements. The limitations of our study are as follows: solely the asteroids in the main belt are considered (i.e., the semimajor axes are in the range from 2 to $4 \mathrm{AU}$ ); solely the three-body resonances with Jupiter and Saturn are taken into account; the resonances are considered in the planar problem, i.e., the longitudes of nodes in the expression for the resonant argument are ignored; the maximum considered order $q_{\max }$ of the three-body resonances is set equal to 6 .

Our project is intended for the resonance analysis of the orbital data presented at the "Asteroids - Dynamic Site" (AstDyS) maintained by A. Milani, Z. Knežević and their coworkers (http://hamilton.dm.unipi.it/cgi-bin/astdys/). We take the orbital data for the analysis from this database. Thus the total set under analysis contains $\approx 250000$ objects.

The basic purpose of our work is to identify the current three-body resonances that all the asteroids from the given set are currently involved in. More specifically, each object from the set should be put in correspondence to a threebody resonance (or none, if there is no resonance). The first attempt of massive identification of asteroids in three-body resonances was made by Nesvorný and Morbidelli (1998): 255 objects were identified to be in three-body resonances. The libration/circulation of the resonant argument for asteroids suspected to reside in the resonances was analyzed visually. In our case the data set is much greater, and therefore the procedure ought to be completely automatic. Besides, here we apply a unified bound on the order. This allows one to construct a homogeneous identification list for a further statistical analysis.

To form a general statistical view of the resonant structure of the main belt, we also accomplish a massive identification of asteroids in two-body resonances with Jupiter, and compare the abundances of asteroids in three-body and twobody resonances.

\section{The identification matrix}

As a first stage of the identification process we build an "identification matrix". It consists of two main columns. The first one contains designations of resonances, and the second one contains the corresponding resonant values of the semimajor axis.

The designations of resonances are given in the notation $m_{\mathrm{J}} m_{\mathrm{S}} m(q)$, where $m_{\mathrm{J}}, m_{\mathrm{S}}, m$ are the integer coefficients in the resonant argument (2), and $q$ is the resonant order, as defined above. The values of $m_{\mathrm{J}}, m_{\mathrm{S}}, m$ are given with their signs. Thus, examples of this notation look like as follows: 5-2-2(1), 2+2-1(3).

We construct a set of the resonant arguments for all possible three-body resonances up to a fixed order $q_{\max }$ in the following way.

We fix the maximum absolute value $M_{\max }$ of each integer $m_{\mathrm{J}}, m_{\mathrm{S}}, m$ to be equal to $q_{\max }+2$, where $q_{\max }$ is the maximum resonant order. It is assumed that 


$$
m_{\mathrm{J}}>0, \quad \operatorname{gcd}\left(m_{\mathrm{J}}, m_{\mathrm{S}}, m\right)=1,
$$

where "gcd" stays for the greatest common divisor. It is set to be equal to 1 to avoid the higher order harmonics with greater multiplicity. The multiplicity is defined as equal to $\operatorname{gcd}\left(m_{\mathrm{J}}, m_{\mathrm{S}}, m\right)$. The harmonics with multiplicity greater than 1 are not discernible in our identification procedure, because their arguments librate simultaneously, though with different amplitudes. (Consider, e.g., such resonances as $4-2-2$ and $8-4-4$. The second one, which has multiplicity equal to 2 , in our procedure is set to be equivalent to the first one.)

Then we search through all possible combinations of $m_{\mathrm{J}}, m_{\mathrm{S}}, m$ and identify those satisfying the D'Alembert rule (3) and our technical restrictions (5) and (6).

Let us demonstrate how the resonant value of the semimajor axis is calculated. According to the definition of the three-body resonance (1), the time derivative $\dot{\sigma}_{p_{\mathrm{J}}, p_{\mathrm{s}}, p}$ should be equal to zero. Let us, following Murray et al. (1998), assume that $\dot{\varpi} \approx 0$, in the first approximation. Then, for the resonant value of mean motion, one has

$$
n_{\text {res }}=-\frac{1}{m}\left(m_{\mathrm{J}} \dot{\lambda_{\mathrm{J}}}+m_{\mathrm{S}} \dot{\lambda} \mathrm{S}+p_{\mathrm{J}} \dot{\varpi}_{\mathrm{J}}+p_{\mathrm{S}} \dot{\varpi}_{\mathrm{S}}\right) .
$$

Using Kepler's third law, one obtains for the resonant semimajor axis

$$
a_{\mathrm{res}}=\left(\frac{k}{n_{\mathrm{res}}}\right)^{2 / 3},
$$

where $n_{\text {res }}$ is given by formula (9), and $k$ is the Gauss constant.

Holman and Murray (1996) and Murray et al. (1998) obtained an approximate formula for the precession rate of an asteroid's orbit:

$$
\dot{\varpi} \approx \frac{\mu}{2 \pi}\left(\frac{a}{a_{\mathrm{J}}}\right)^{1 / 2} \varepsilon^{2} n_{\mathrm{J}},
$$

where $\mu \approx 1 / 1047$ is the mass of Jupiter in units of the mass of the Sun, $n_{\mathrm{J}}$ and $a_{\mathrm{J}}$ are Jupiter's mean motion and the semimajor axis of Jupiter's orbit, respectively, and

$$
\varepsilon=\frac{a_{\mathrm{J}}-a}{a_{\mathrm{J}}} .
$$

For $a$, we substitute here the resonant value of the semimajor axis as given by Eq. (10). Thus the value of $\dot{\varpi}$ is calculated. Iterating, one obtains an adequate value of $a_{\text {res }}$. The second primary column of the identification matrix is filled with the resonant values of the semimajor axes, calculated as described, with the accuracy of no less than $10^{-3}$ AU. This accuracy far exceeds the necessary one, because we check the asteroids for belonging to a given resonance in a far greater neighborhood $\left( \pm 10^{-2} \mathrm{AU}\right)$ of the computed resonant value of $a_{\text {res }}$.

Nesvorný and Morbidelli (1999) calculated $a_{\text {res }}$ of the leading subresonances (i.e., of the multiplet components $\sigma_{0,0,-m_{\mathrm{J}}-m_{\mathrm{S}}-m}$ ) of 19 three-body resonances 
with the accuracy of $10^{-4} \mathrm{AU}$ : they equated the time derivative of Eq. (2) to zero, and, using the values of $\dot{\lambda}_{\mathrm{J}}$ and $\dot{\lambda}_{\mathrm{S}}$ as given by Bretagnon (1990) and $\varpi$ as found using the code by Milani and Knežević (1994), calculated $\dot{\lambda}$. All values of $a_{\text {res }}$ given in (Nesvorný and Morbidelli, 1999, table 1) agree quite closely with the corresponding values of $a_{\text {res }}$ calculated here iteratively, as described above, for our matrix. The agreement is illustrated in Table 1, where an extract from the identification matrix is presented.

Table 1: An extract from the identification matrix

\begin{tabular}{rrrccc}
\hline$m_{\mathrm{J}}$ & $m_{\mathrm{S}}$ & $m$ & $q$ & $\begin{array}{c}a_{\text {res }}(\mathrm{AU}) \\
\text { (this study) }\end{array}$ & $\begin{array}{c}a_{\text {res }}(\mathrm{AU}) \\
\text { (Nesvorný and Morbidelli, 1999) }\end{array}$ \\
\hline 2 & 3 & -1 & 4 & 2.3912 & - \\
4 & -2 & -1 & 1 & 2.3978 & 2.3977 \\
2 & 2 & -1 & 3 & 2.6148 & 2.6155 \\
4 & -3 & -1 & 0 & 2.6232 & 2.6229 \\
1 & 4 & -1 & 4 & 2.7432 & - \\
3 & -1 & -1 & 1 & 2.7527 & 2.7525 \\
1 & 3 & -1 & 4 & 3.0673 & - \\
3 & -2 & -1 & 0 & 3.0798 & 3.0790 \\
5 & -7 & -1 & 3 & 3.0925 & - \\
5 & -2 & -2 & 1 & 3.1744 & 3.1751 \\
\hline
\end{tabular}

\section{Dynamical identification}

We use the following procedure of dynamical identification.

First of all, each asteroid's orbit from the adopted set of 249567 objects is computed for $10^{5} \mathrm{yr}$. The perturbations from all planets (from Mercury to Neptune) and Pluto are taken into account. The hybrid integrator of mercury6 package (Chambers, 1999) is used. In some cases the orbit9 integrator (http://adams.dm.unipi.it/ orbmaint/orbfit/) is used as well, to verify the results. The computed trajectories are kept in files for further usage. The trajectories are output with the time step of $1 \mathrm{yr}$.

After integration is over, the objects are taken from the adopted set, and the mean value of the semimajor axis is computed for each object. Using this value, a set of preliminary resonant arguments $\sigma_{\text {res }}$ is found in the identification matrix. Each argument $\sigma_{\text {res }}$ is then analyzed on the presence of libration/circulation, using the computed trajectory of the object.

We distinguish two types of resonant libration: pure and transient. By definition, the libration is pure, if it lasts during the whole time interval of integration, i.e., $10^{5}$ yr. An example of such libration is given in Fig. 1, where the time behaviour of the resonant argument, alongside with the orbital elements, is demonstrated for asteroid 463 Lola, resonance $4-2-1$. 
The libration is defined as transient, if circulation appears at any time during this interval. An example is given in Fig. 2; this is the case of asteroid 490 Veritas, resonance $5-2-2$. In Fig. 2, it may seem unusual that Veritas exhibits circulation of the resonant argument while the semimajor axis remains almost constant (especially in comparison with Fig. 9, which is considered below and which shows the orbital elements and resonant argument of 1915 Quetzálcoatl, residing in the two-body transient resonance $3 / 1$ ). This difference is explained by a large difference in the widths of the resonances: e.g., at $e=0.1$ the width of resonance $3 / 1$ is $\sim 5$ times greater than that of resonance $5-2-2$ (see fig. 1 in Morbidelli and Nesvorný 1999). Moreover, 1915 Quetzálcoatl has a very large eccentricity $(\sim 0.6-0.8)$. At $e=0.1$, the half-width of the $5-2-2$ resonance is $\approx 0.002 \mathrm{AU}$ (see table 1 in Nesvorný and Morbidelli 1999 and fig. 1 in Morbidelli and Nesvorný 1999). For Veritas, the eccentricity is $\sim 0.06$ (see Fig. 2), hence the half-width is even smaller. This makes the shift in $a$, when $\sigma$ is circulating, almost imperceptible, especially in the digitally unfiltered $a$. When $a$ is filtered, such shifts look more obvious; see fig. 2 in (Knežević et al., 2002) and fig. 3 in (Tsiganis et al., 2007). Note that, when the circulations are short-term, the shifts in $a$ can be imperceptible even in the digitally filtered element: see fig. 1 in (Nesvorný and Morbidelli, 1998), where the time behaviour of Veritas in the digitally filtered elements is shown.

To distinguish between transient-resonant and non-resonant behaviours we introduce a technical parameter: the resonance minimum time, which we set to be equal to $20000 \mathrm{yr}$. Roughly speaking, this is the minimum time interval of resonant librations, for an asteroid to be regarded as resonant. An exact definition is given below.

Nesvorný and Morbidelli (1998) identified resonant behaviour visually and used more subjective criteria, according to which the asteroid is resonant if "(1) the corresponding resonant angle shows evident librations during the integration time span or (2) the resonant angle circulates with a period longer than several thousand years" (Nesvorný and Morbidelli, 1998). In fact the second criterion threshold is analogous to our resonance minimum time, because our automatic procedure measures the time intervals of libration as such when no circulation is present, as described in detail below. Our criterion threshold, being larger, is more restrictive; however, in both cases the threshold is much greater than the timescales of circulation far from resonance; the latter timescales are of the order of asteroidal orbital periods.

The complete procedure of identification is as following. When computing each trajectory, the resonant argument value for the guiding subresonance is calculated at each step in time, and these values are written in a file, in function of time. After the trajectory computation is over, the time behaviour of the resonant argument is analyzed. If a current value of the resonant argument is different from its previous value by more than $2 \pi$, this means that there is a break, which takes place if there is either circulation or apocentric libration. To distinguish between these two cases, the same procedure is repeated, but with an artificial shift of the resonant argument. This shift is equal to $\pi$. Then, if there is apocentric libration, it turns into pericentric one, and there are no breaks. 
At the beginning of the trajectory analysis, two variables are initiated: the first one is the duration of current libration, and the second one is the total time of libration. If circulation whenever starts, the first variable value is added to the second one and the first variable is reset to zero. At the end of the analysis, it is checked whether the second variable (total time of libration) is equal to the full time of computation $\left(10^{5} \mathrm{yr}\right)$. If yes, then the asteroid is regarded to be in pure resonance. If no, but the second variable value exceeds the resonance minimum time, then the asteroid is in transient resonance. If the second variable value is less than the resonance minimum time, then the asteroid is regarded to be non-resonant. The adopted pure/transient division of resonances is thus mostly technical, because it depends on the chosen time interval of computation.

We use solely the direct method of identification, i.e., the resonant argument is checked on the subject of libration. Any secondary and/or auxiliary criteria, such as the semimajor axis behaviour, the location of objects in the proper "semimajor axis - eccentricity" plane, the Lyapunov exponents are left for future explorations, — with a partial exception in the latter case, see Section 5.

In our identification procedure, it is formally possible that an asteroid might be identified as belonging to two or even more resonances, because all resonances in a rather broad neighbourhood in semimajor axis $\left( \pm 10^{-2} \mathrm{AU}\right.$, as mentioned above) in the identification matrix are scanned on the subject of libration. If two three-body resonances are close enough in semimajor axis, and especially if they also overlap with a strong two-body resonance, an asteroid may intermittently diffuse from one to another resonance; a small number of such objects have been identified, as belonging to two resonances. In our lists, each object of this kind has been finally attributed to the resonance where it stayed for a longer time, to avoid complication of statistics. Analysis of such objects (we call them "rogue-resonant" asteroids) will be given elsewhere.

Besides, since the data used is unfiltered, some misidentifications may take place when the libration amplitude is high. To estimate the formal statistical error of our procedure, we randomly chose 300 asteroids identified as pureresonant and visually checked the libration of their resonant arguments. It turned out that only 4 objects $(1.3 \%$ of the set) were misidentified. Thus the statistical error of the procedure is $\approx 1 \%$.

All identified pure three-body-resonant asteroids, grouped according to association to a given resonance, are listed in the Appendix A. The top ten resonances, that are most "populated", are listed in Table 2. The last column of Table 2 contains analytical estimates of the resonance width (at the asteroidal eccentricity $e=0.1$ ), according to (Nesvorný and Morbidelli, 1999, table 1). One can see that, generally, broader the resonance, greater is the number of objects residing in it. However, this tendency is not strict. The reason is that the dynamics here is strongly interrelated with physics: e.g., a collisional disintegration of an asteroid can strongly increase abundance of objects in a particular resonance, thus disturbing the expected correlation.

For each resonance, statistics on the asteroids in pure and transient librations have been calculated. The statistical results are summarized in Table 3 . The fraction of asteroids in three-body resonances (transient plus pure) turns out to 

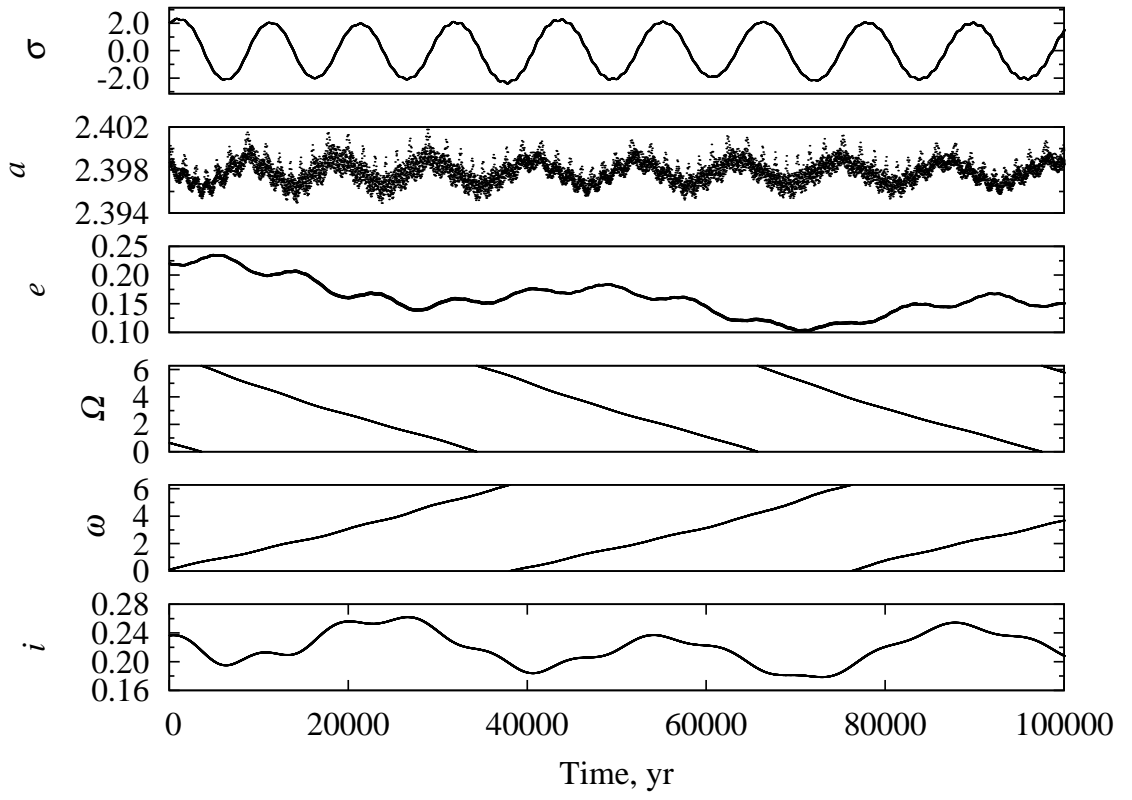

Figure 1: The orbital elements and resonant argument of 463 Lola. Resonance $4-2-1$.

be $\approx 4.4 \%$ of the whole set. This is rather close to the value $4.6 \%(\approx 1500$ in the set of $\approx 32400$ ) found by Nesvorný and Morbidelli (1998) to serve as a lower bound for the relative number of resonant asteroids. As follows from Table 3, the fraction of asteroids in pure three-body resonances turns out to be $\approx 0.94 \%$ of the whole set.

The third column of Table 3 contains a prediction for the numbers of asteroids residing in resonances of all orders; this subject is discussed in the next Section.

Let us consider the location of three-body-resonant asteroids in the "semimajor axis - eccentricity" plane. For each asteroid in a given resonance we calculate the average values (over the whole time interval of integration) of the semimajor axis and eccentricity and plot these values in the " $a-e$ " plane. We have accomplished this procedure for each resonance with known structure of the resonant multiplet, as calculated by Nesvorný and Morbidelli (1999), so that the separatrix of the leading subresonance could be drawn. An example of such a plot is given in Fig. 3. It is clear that the percentage of "outliers" (objects out of the separatrix cell) is zero. This confirms the good accuracy of the accomplished identification procedure in the case of this particular resonance. A detailed study of the " $a-e$ " plots will be given elsewhere.

We have cross-checked the lists of resonant objects identified in our study and in (Nesvorný and Morbidelli, 1998). It turns out that the number of resonant 


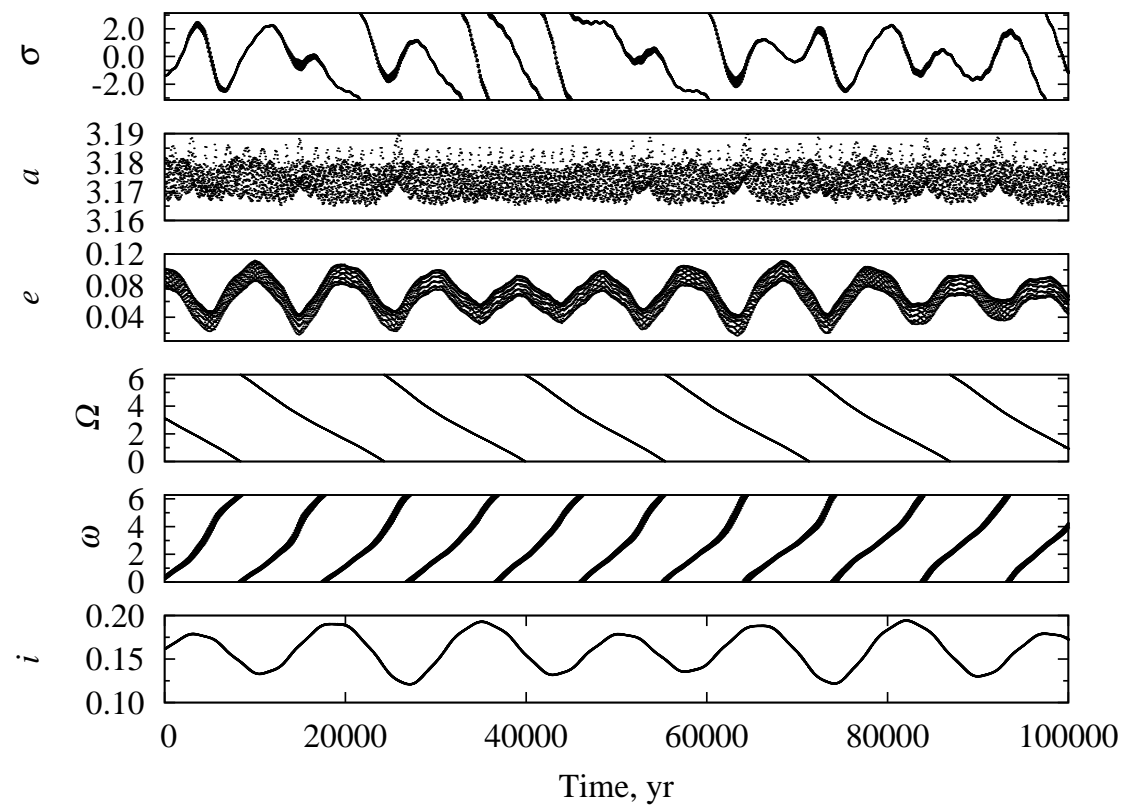

Figure 2: The orbital elements and resonant argument of 490 Veritas. Resonance $5-2-2$.

objects listed by Nesvorný and Morbidelli (1998) but not identified as resonant in our study does not exceed 1\% of the list of Nesvorný and Morbidelli (1998). This confirms that the differences in the methodologies of identification in the two studies do not play any significant role in what concerns the reliability of results.

\section{Expected abundances of asteroids in high- order three-body resonances}

The obtained list of resonant asteroids is obviously not complete, due to the limitations of identification criteria. First of all, the resonant order $q \leq 6$. Presumably, there is a lot of objects in resonances of higher orders. To take account of them, let us analyze the asteroid distribution in the resonant order $q$. The necessary data as derived from the results of the identification procedure are presented in Table 4.

The constructed differential distributions (histograms) in $q$ are shown in Fig. 4 for the case of transient plus pure resonances, and in Fig. 5 for the case of pure resonances.

As an approximating function, we have chosen the power law 


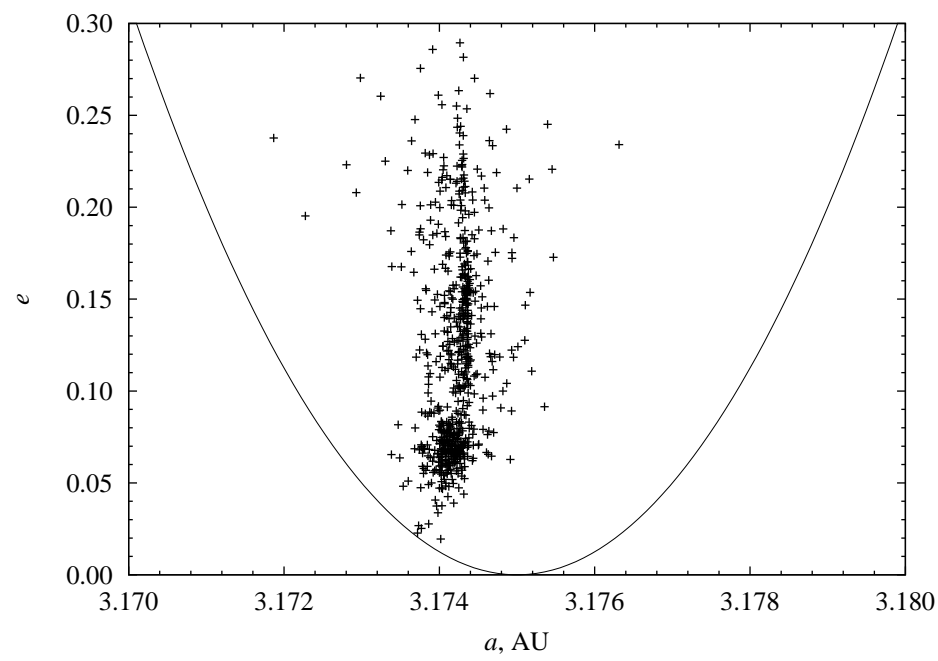

Figure 3: The asteroids identified to be in resonance $5-2-2$ : location in the " $a-e$ " plane. Solid curve: the separatrix of the leading subresonance, as calculated in (Nesvorný and Morbidelli, 1999).

Table 2: The top ten most populated three-body resonances Sun-Jupiterasteroid

\begin{tabular}{crccccc}
\hline$m_{\mathrm{J}}$ & $m_{\mathrm{S}}$ & $m$ & $\begin{array}{c}a_{\text {res }} \\
(\mathrm{AU})\end{array}$ & $\begin{array}{c}\text { Trans. + pure, } \\
\text { number of objects }\end{array}$ & $\begin{array}{c}\text { Pure, number } \\
\text { of objects }\end{array}$ & $\begin{array}{c}\text { Res. width (AU) at } e=0.1 \\
\text { (Nesvorný and Morbidelli, 1999) }\end{array}$ \\
\hline 5 & -2 & -2 & 3.1744 & 699 & 182 & 0.0056 \\
4 & -2 & -1 & 2.3978 & 688 & 595 & 0.0024 \\
3 & -2 & -1 & 3.0798 & 621 & 134 & 0.0045 \\
3 & -1 & -1 & 2.7527 & 540 & 203 & 0.0019 \\
2 & 2 & -1 & 2.6148 & 470 & 34 & 0.00015 \\
4 & -3 & -1 & 2.6232 & 455 & 90 & - \\
2 & 3 & -1 & 2.3912 & 343 & 56 & - \\
5 & -7 & -1 & 3.0925 & 314 & 48 & - \\
1 & 3 & -1 & 3.0673 & 300 & 52 & - \\
1 & 4 & -1 & 2.7432 & 284 & 47 & - \\
\hline
\end{tabular}

$$
N=a q^{b},
$$

where $a$ and $b$ are two fitting parameters; $b<0$. We have also tried the exponential law $N \propto \exp (c q)$ (where $c<0$ ), but it has turned out to be inappropriate, the statistical significance of fitting being very low.

Since we are interested in the tail behaviour of the distributions, we have 
Table 3: Asteroids in three-body resonances, statistics

\begin{tabular}{lrrr}
\hline & NM98* $^{*}$ & $\begin{array}{r}\text { This study, } \\
q_{\max }=6\end{array}$ & $\begin{array}{r}\text { This study, prediction } \\
\text { for } q_{\max }=\infty\end{array}$ \\
\hline The whole set of objects & 5400 & 249567 & 249567 \\
Objects with integrated orbits & 836 & 249567 & 249567 \\
Transient+pure-resonant objects & 255 & 11039 & - \\
The same, fraction of the studied set & $4.7 \%$ & $4.4 \%$ & - \\
Pure-resonant objects & - & 2338 & 2854 \\
The same, fraction of the studied set & - & $0.94 \%$ & $1.1 \%$ \\
\hline
\end{tabular}

*Nesvorný and Morbidelli (1998).

Table 4: Abundances of asteroids in three-body resonances in function of $q$

\begin{tabular}{lrrrrrrr}
\hline$q$ & 0 & 1 & 2 & 3 & 4 & 5 & 6 \\
\hline Transient+pure & 1371 & 2884 & 1367 & 2256 & 1394 & 997 & 770 \\
Pure & 356 & 1119 & 194 & 251 & 214 & 117 & 87 \\
\hline
\end{tabular}

used the data for $q \geq 1$, ignoring the specific case $q=0$. In the case of all resonant (transient-resonant plus pure-resonant) asteroids (see Fig. 4) we find $a=2821 \pm 454, b=-0.57 \pm 0.18$; and the correlation coefficient $R^{2}=0.71$. As soon as $|b|<1$, the predicted number of asteroids in high-order resonances is formally infinite; in practice this means that it can comprise up to $\sim 100 \%$ of the whole set.

In the case of pure-resonant asteroids (see Fig. 5) we find $a=1095 \pm 103$, $b=-1.64 \pm 0.28 ; R^{2}=0.94$. As soon as $|b|>1$, the predicted total number of asteroids in high-order resonances is finite. Using Eq. (13), the number of objects with $q \geq 7$ in the studied set is estimated to be equal to $\approx 516$, or $\approx 22.1 \%$ of the identified number (2338). Therefore, the predicted total number of asteroids in pure three-body resonances is estimated as 2854, constituting $1.1 \%$ of the whole set (249567).

A note of caution is in order here. One has to admit that the fits made in this Section are based on few points, and, therefore, any statistical predictions, made with these formulas, are uncertain. What is more, one cannot even expect to find smooth distributions and/or strict correlations in this field of research, where dynamics is strongly interrelated with physics: the abundances of objects in resonant groups are regulated by various processes, e.g., by collisions and the Yarkovsky effect. Interactions with asteroidal families are important. Of course, higher order resonances should be directly analyzed in the future.

Another complicating factor is that the subresonance overlap (and hence, the degree of chaoticity) in the multiplets is expected to decrease asymptotically at $q \gg 1$. The reason is that the subresonance typical width scales with 


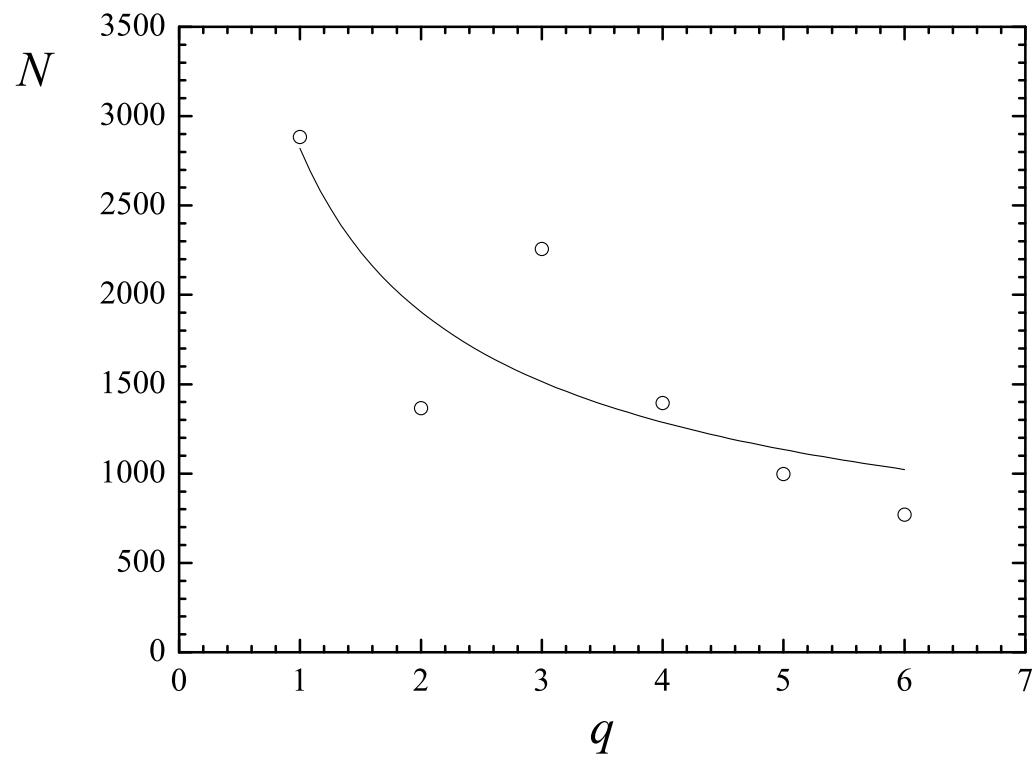

Figure 4: Circles: the distribution of all resonant (transient-resonant plus pureresonant) asteroids in resonant order $q \geq 1$. Solid curve: the power-law fitting.

$q$ as $\sim e^{q}$ (where $e$ is the eccentricity), whereas the subresonances separation (determining the multiplet width) remains basically constant, depending solely on the secular precession rates of the pericentres; thus the overlap/interaction of subresonances in the multiplets decreases asymptotically at $q \gg 1$ (Nesvorný and Morbidelli, 1999, p. 268-269). When the ratio of the subresonances separation to the width of the leading subresonance is much greater than one, the separatrix chaotic layers are exponentially thin with this ratio, see (Chirikov, 1979; Shevchenko, 2008, 2011). It is straightforward to suppose that, if one fixes the asteroidal eccentricity, three-body resonances with increasing order become basically regular (Nesvorný and Morbidelli, 1999). Thus, if the resonances are populated uniformly in the eccentricity, the power-law extrapolation for the high-order transient-resonant populations fails beyond some order. Only if this critical order is high enough, the extrapolation-based estimates might be appropriate. 


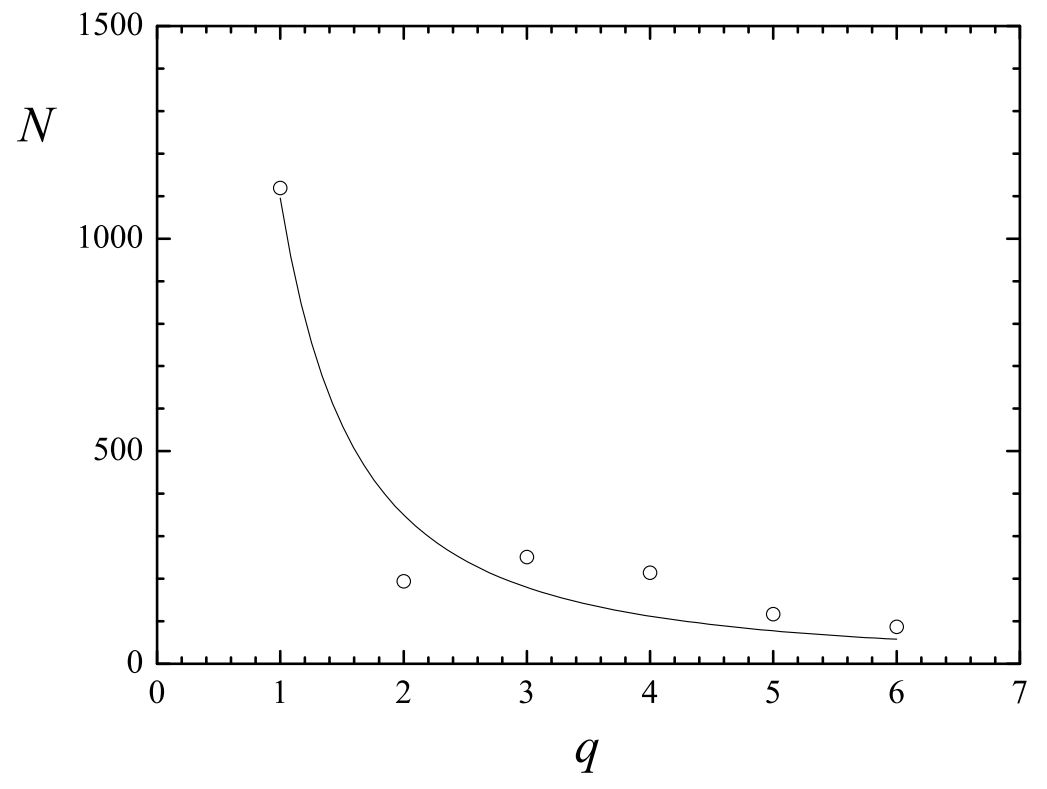

Figure 5: Circles: the distribution of pure-resonant asteroids in resonant order $q \geq 1$. Solid curve: the power-law fitting.

\section{Lyapunov exponents in three-body reso- nances}

The AstDyS database provides information on the maximum Lyapunov exponents for almost all asteroids contained in it. The provided values are computed on time intervals of $2 \mathrm{mln}$ yr, i.e., on time intervals 20 times greater than that we use in our identification procedure. It is instructive to check how the data on Lyapunov exponents correlate with the pure/transient division (adopted in our study) of the resonant asteroids.

In the transient case the motion is expected to be chaotic, and in the pure case to be essentially regular, because transitions from libration to circulation and vice versa are inevitable in a separatrix chaotic layer of resonance. However, the adopted relatively short integration time may imply that many "pure"resonant asteroids become transient on longer timescales.

First of all, let us build distributions of resonant asteroids in $L$ (the maximum Lyapunov exponent). The constructed differential distributions (histograms) are presented in a single plot in Fig. 6. The Lyapunov exponents are given in units of $(\mathrm{mln} \mathrm{yr})^{-1}$. (Note that in the AstDyS database they are given in 
units of $\mathrm{yr}^{-1}$.) $N$ is the number of objects in the interval $(L, L+\Delta L)$, where $\Delta L=10$. The grey histogram is for the asteroids in transient resonances, and the black one is for the asteroids in pure resonances. The histograms are cut off at $L=500$, because the objects with greater values of $L$ are rare; they are all transients.

From Fig. 6 it is clear that the transients have a much more extended distribution in $L$, in comparison with the pure-resonant objects, as expected. This difference is uniform in all resonant groups, as can be directly seen from Fig. 7, where the maximum Lyapunov exponents are plotted versus the semimajor axis $a$ : the positions of open dots (representing transients) extend to much greater heights, in comparison with the pure-resonant objects, in all resonant groups. Note that this plot is built without any cut-off in $L$, i.e., all objects are shown. It is evident that only transients have very large Lyapunov exponents, corresponding to Lyapunov times as small as $\approx 570 \mathrm{yr}$.

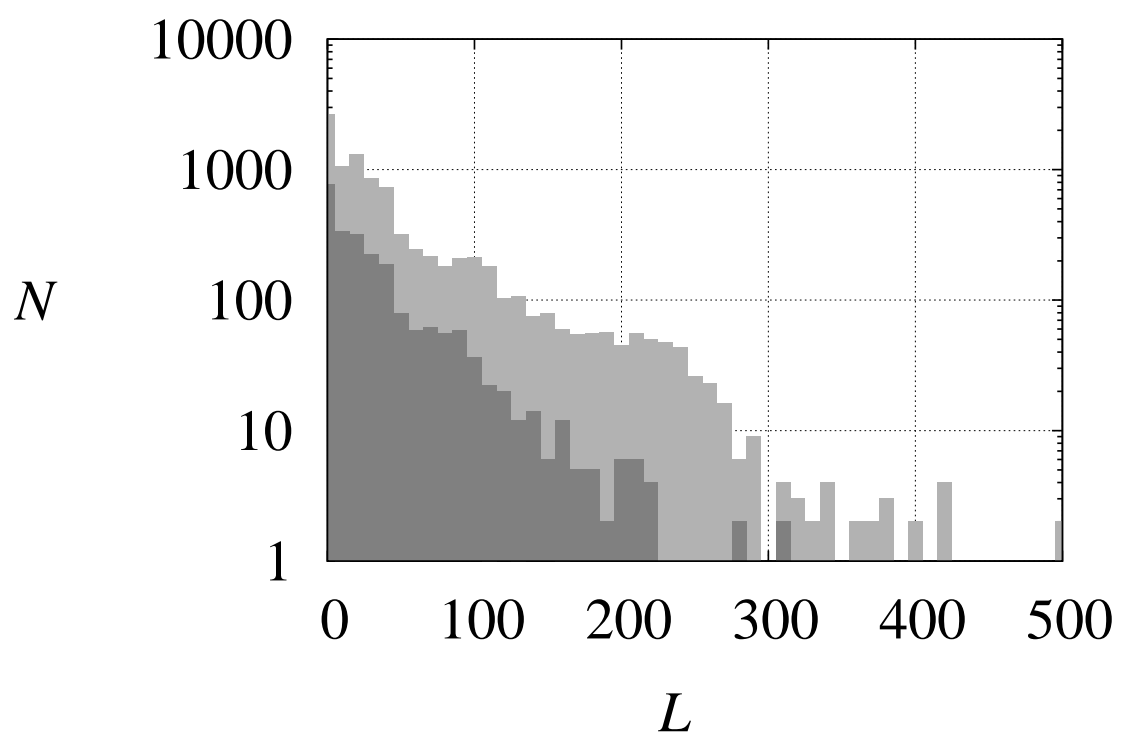

Figure 6: The distributions of resonant asteroids in the value of maximum Lyapunov exponent. Grey: asteroids in transient resonances. Black: asteroids in pure resonances.

Among all identified pure-resonant objects, the average $L$ is $34.3(\mathrm{mln} \mathrm{yr})^{-1}$, and among all identified transients it is $49.7(\mathrm{mln} \mathrm{yr})^{-1}$. The Lyapunov times are $\approx 29200$ and $\approx 20100 \mathrm{yr}$, respectively. Among all objects of the AstDyS database with measured Lyapunov exponents, the average $L=20.8(\mathrm{mln} y \mathrm{r})^{-1}$, corresponding to the Lyapunov time $\approx 48100 \mathrm{yr}$. Thus both pure-resonant and transient-resonant objects turn out to be more chaotic than a typical asteroid. 


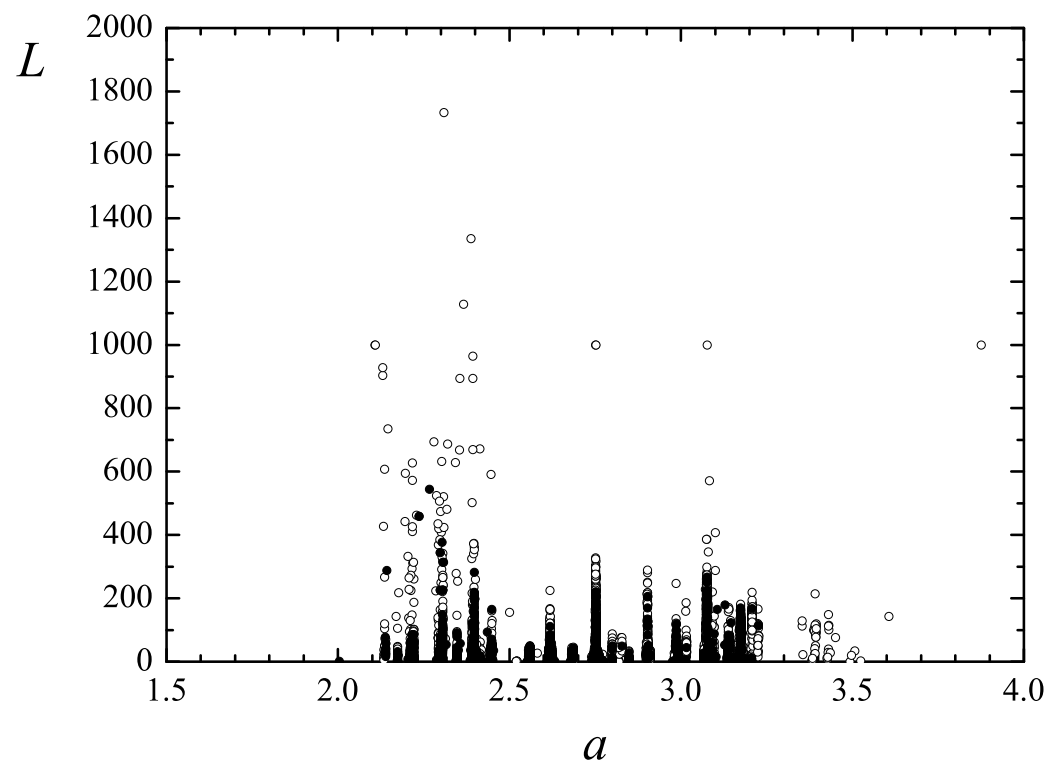

Figure 7: The maximum Lyapunov exponents versus the semimajor axis $a$. Open dots: asteroids in transient resonances. Black dots: asteroids in pure resonances.

The fact that many pure-resonant asteroids have definitely non-zero Lyapunov exponents signifies that they become transient on the timescales longer than the adopted integration time $\left(10^{5} \mathrm{yr}\right)$. This underlines the conditional character of the "pure/transient" technical classification adopted in our study.

\section{Two-body resonances with Jupiter}

To form a more general statistical view of the actual resonant structure of the main belt, it is instructive to compare the abundances of asteroids in three-body resonances with the abundances of asteroids in two-body resonances. For this purpose, we have performed a procedure of identification of asteroids in twobody resonances with Jupiter, analogous to that described above for the case of three-body resonances. In this identification procedure, we assume circular and zero inclination orbits of perturbing planets.

Taking into account the D'Alembert rule, the resonant argument for the resonance of order $q$ is defined by the following formula (Murray and Dermott, 1999; Morbidelli, 2002; Gallardo, 2006): 


$$
\sigma=(p+q) \lambda_{\mathrm{J}}-p \lambda-q \varpi,
$$

where $\lambda_{\mathrm{J}}$ and $\lambda$ are the mean longitudes of Jupiter and an asteroid, respectively, and $\varpi$ is the longitude of perihelion of the asteroid; $q$ is the resonant order, $p$ is integer.

Of course, this setting of the identification problem is a rather simplified one. When real resonant asteroids are considered, the "circular" subresonance term is not expected to dominate universally; what is more, the resonant asteroid might reside in another component of a multiplet (see Holman and Murray 1996; Murray and Holman 1997). We regard the adopted approach as a first approximation, and leave considerations of all possible subresonances for the future.

The resonant value of the semimajor axis of an asteroidal orbit is given by

$$
a_{\mathrm{res}} \approx a_{\mathrm{J}}(1+\mu)^{-1 / 3}\left(\frac{p}{p+q}\right)^{2 / 3}
$$

where $a_{\mathrm{J}}$ is the semimajor axis of Jupiter's orbit, and $\mu$ is the mass of Jupiter in units of the mass of the Sun (see, e.g., Murray and Dermott 1999; Gallardo 2006).

The identification matrix is constructed in the way analogous to that described in Section 2. Similar to the case of three-body resonances, it is assumed that $\operatorname{gcd}(p, q)=1$. Besides, $1 \leq p \leq 11$.

The resonant order range is limited to $0 \leq q \leq 9$. Recall that in the case of three-body resonances we have set $0 \leq q \leq 6$. Choosing the upper bounds with the difference equal to 3 allows one to adequately compare identification statistics for two-body and three-body resonances, because "a three-body resonance of a given order $q$ should have roughly the same strength as a usual resonance of order $q+3$ for eccentricity of about 0.05-0.10" (Nesvorný and Morbidelli, 1998). Such a trick was used in Nesvorný and Morbidelli (1998) for similar purposes.

Figs. 8 and 9 show the time behaviour of the resonant argument and orbital elements of 1915 Quetzálcoatl and 190 Ismene, as two typical examples. The top ten most "populated" resonances, identified in our study, are listed in Table 5. All identified pure two-body-resonant asteroids, grouped according to association to a given resonance, are listed in the Appendix B. ${ }^{1}$

The resulting statistics of identified objects are listed in Tables 6 and 7. As follows from Table 6 , a half of all identified asteroids in pure two-body resonances are Trojans $(1669 / 3132 \approx 53 \%)$. Pure Trojans plus pure Hildas constitute $\approx 85 \%$ of all asteroids in pure two-body resonances in our set. In Table 7 , the $q$ dependence of the resonant asteroid abundances is presented. The dependence is obviously irregular and does not permit any smooth decay approximation. Especially one should point out the negligible asteroid abundances at $q=3$ and $q=9$.

\footnotetext{
${ }^{1}$ One may wonder why 279 Thule is absent in the $4 / 3$ entry. The matter is that our automatic procedure identifies it as being in transient resonance, not in pure one, because the resonant argument sometimes goes out of the range $(-\pi,+\pi)$.
} 


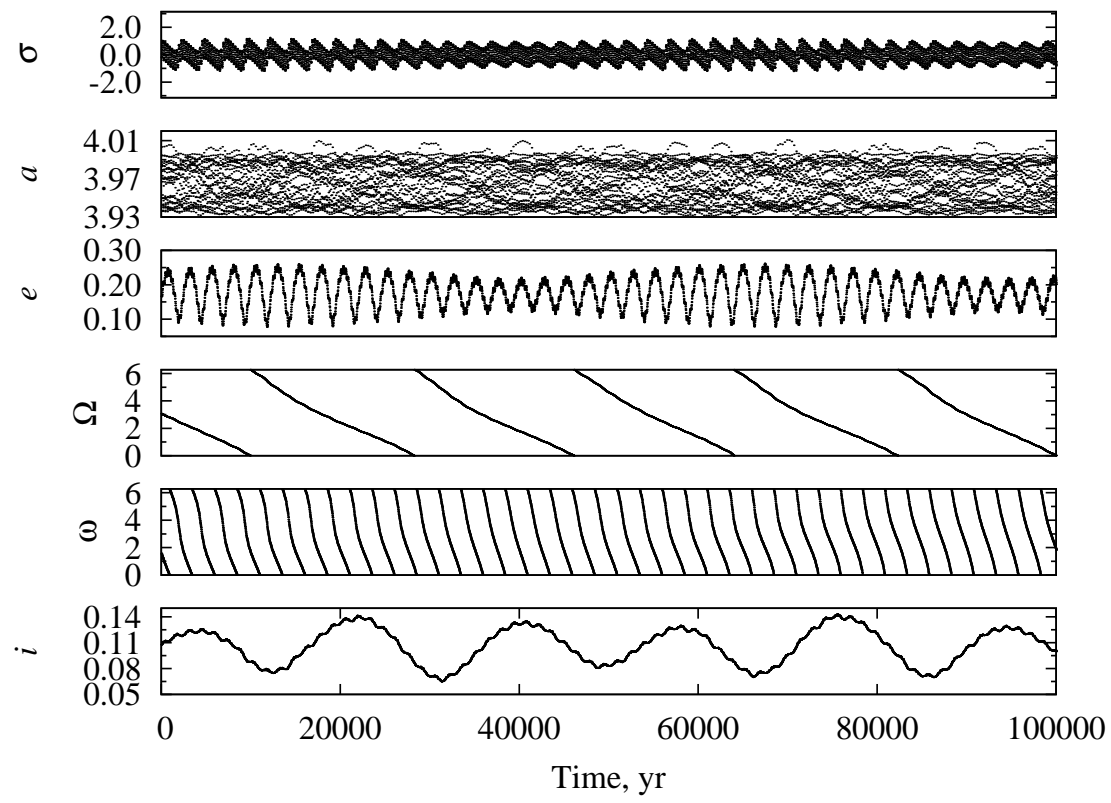

Figure 8: The orbital elements and resonant argument of 190 Ismene. Pure resonance $3 / 2$.

Table 5: The top ten most populated two-body resonances with Jupiter. The main belt and Trojans

\begin{tabular}{rrccrrl}
\hline$p+q$ & $p$ & $q$ & $\begin{array}{c}a_{\text {res }} \\
(\mathrm{AU})\end{array}$ & $\begin{array}{r}\text { Transient } \\
+ \text { pure }\end{array}$ & Pure & Notes \\
\hline 1 & 1 & 0 & 5.2043 & 1670 & 1669 & Trojan swarms \\
3 & 2 & 1 & 3.9716 & 1033 & 1007 & Hilda group \\
11 & 5 & 6 & 3.0766 & 351 & 49 & \\
2 & 1 & 1 & 3.2785 & 274 & 227 & Griqua family \\
8 & 3 & 5 & 2.7063 & 232 & 20 & \\
7 & 2 & 5 & 2.2576 & 208 & 20 & \\
10 & 3 & 7 & 2.3322 & 165 & 15 & \\
11 & 4 & 7 & 2.6514 & 165 & 0 & \\
9 & 4 & 5 & 3.0309 & 163 & 55 & \\
3 & 1 & 2 & 2.5020 & 63 & 32 & Alinda family \\
7 & 3 & 4 & 2.9583 & 51 & 17 & \\
\hline
\end{tabular}

It turns out that in transient plus pure resonances the asteroids are $\approx 2.5$ times more abundant in three-body resonances than in two-body resonances (11039 versus 4450); and in pure resonances the abundances are in the ratio 


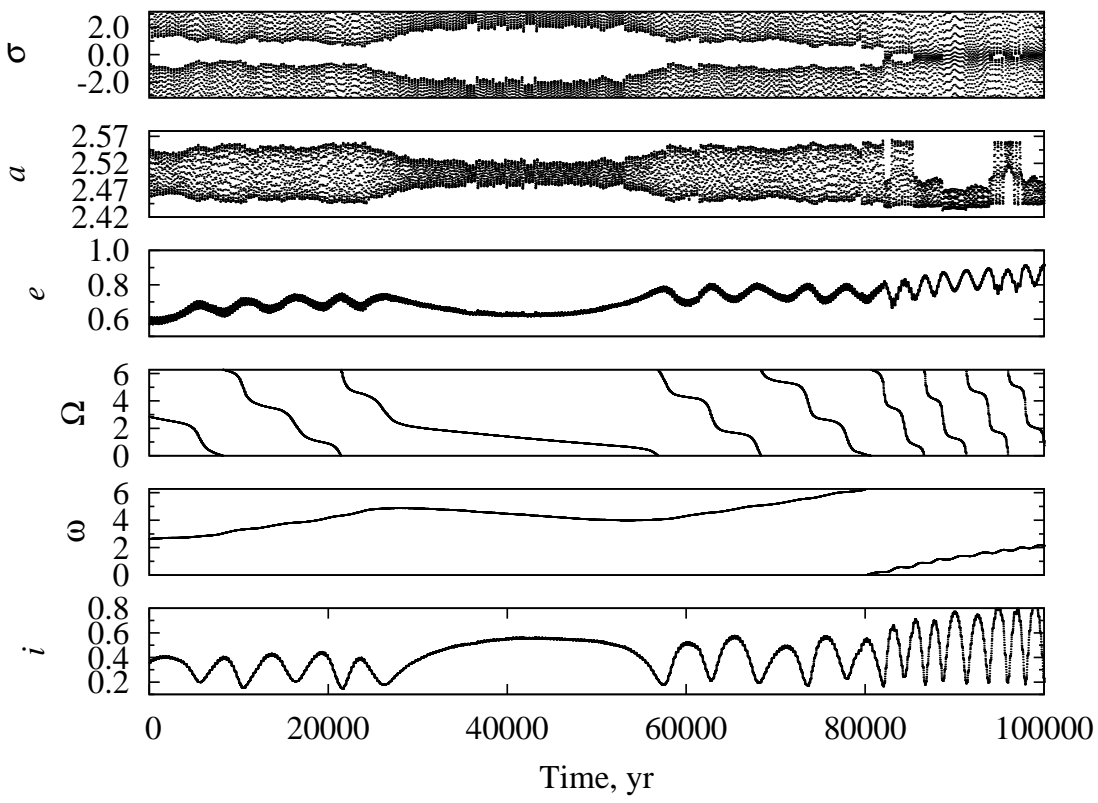

Figure 9: The orbital elements and resonant argument of 1915 Quetzálcoatl. Transient resonance $3 / 1$.

Table 6: Asteroids in two-body resonances with Jupiter, statistics. The main belt and Trojans

\begin{tabular}{lcc}
\hline & $\begin{array}{c}\text { Number } \\
\text { of resonances }\end{array}$ & $\begin{array}{c}\text { Number } \\
\text { of objects }\end{array}$ \\
\hline Transient + pure & 21 & $4450(1.78 \%)$ \\
Pure & 16 & $3132(1.25 \%)$ \\
\hline
\end{tabular}

Table 7: Abundances of asteroids in two-body resonances in function of $q$

\begin{tabular}{lrrrrrrrrrr}
\hline$q$ & 0 & 1 & 2 & 3 & 4 & 5 & 6 & 7 & 8 & 9 \\
\hline Transient + pure & 1670 & 1310 & 63 & 15 & 57 & 603 & 352 & 342 & 37 & 1 \\
Pure & 1669 & 1236 & 32 & 5 & 19 & 95 & 49 & 23 & 4 & 0 \\
\hline
\end{tabular}

$\approx 3: 4$ (2338 versus 3132). However, if one excludes Trojans and Hildas, the abundance of three-body-resonant asteroids becomes overwhelming: in the case of transient plus pure resonances the ratio of observed abundances of three-bodyresonant objects and two-body-resonant objects becomes equal to $11039 / 1747 \approx$ 6.3 ; and in the case of pure resonances the ratio becomes equal to $2338 / 456 \approx$ 
5.1 .

If Trojans and Hildas are not excluded, the fraction of pure-resonant asteroids among the transient+pure-resonant asteroids is $\approx 3.3$ times greater in the two-body case than in the three-body one $(\approx 70.4 \%$ versus $\approx 21.2 \%)$; thus it might seem that the two-body resonances are much more "pure" on average. However, if one excludes Trojans and Hildas, the fractions become comparable: $26.1 \%$ versus $21.2 \%$.

One should outline that the comparative analysis given here is merely statistical and, thus, formal. However, such an analysis might provide a necessary preliminary stage for a deeper study of the comparative role of two-body and three-body resonances; such a study should comprise consideration of the physical (collisions and the Yarkovsky effect) and dynamical (transport and diffusion) processes, which lead to populating or depopulating the resonances.

\section{Conclusions}

1. We have identified the resonant objects (the objects residing in three-body resonances with Jupiter and Saturn in the main asteroid belt) in the set of all numbered asteroids in the AstDyS database. This set comprises 249567 asteroids catalogued up to the date of April, 2011. The list of all asteroids identified as residing in pure three-body resonances is given in Appendix A.

2. The fraction of asteroids in three-body resonances (transient plus pure) up to the 6 th order inclusive turns out to be $\approx 4.4 \%$ of the total studied set of 249567 asteroids. The fraction of asteroids in pure three-body resonances of the same orders turns out to be $\approx 0.94 \%$ of the total studied set.

3. The top three most populated three-body resonances are: $5-2-2$ (containing 699 transient+pure-resonant asteroids), 4 -2 -1 (688 transient+pureresonant asteroids), $3-2-1$ (621 transient+pure-resonant asteroids). For the pure-resonant asteroids, the "top three" resonances are: $4-2-1,3-1$ -1 , and $5-2-2$, containing 595,203 , and 182 objects, respectively.

4. Using a high-order extrapolation (in the form of a power law) of the $q$ dependence of the number of identified resonant objects, the actual total fraction of asteroids in pure three-body resonances of all orders is estimated as $\approx 1.1 \%$ of the whole set. In what concerns the case of transient three-body resonances, the situation is much less certain, because the power-law extrapolation diverges.

5. We have also identified all objects residing in two-body resonances (of order $0 \leq q \leq 9$ ) with Jupiter in the main asteroid belt, taking the same database of asteroids. The list of all asteroids identified as residing in pure two-body resonances is given in Appendix B. 
6. The half of all identified asteroids in pure two-body resonances are Trojans $(\approx 53 \%)$. The pure Trojans plus pure Hildas constitute $\approx 85 \%$ of all asteroids in the pure two-body resonances. The $q$ dependence of the two-body resonant abundances is clearly irregular and does not permit any smooth decay approximation. Especially one should point out the negligible asteroidal abundances at $q=3$ and $q=9$.

7. In the transient plus pure resonances, the identified asteroids are $\approx 2.5$ times more abundant in the three-body resonances than in the two-body resonances; and in the pure resonances the abundances are comparable. However, if one excludes Trojans and Hildas, the abundance of three-bodyresonant asteroids becomes overwhelming. What is more, taking into account extrapolated abundances in high-order resonances may substantially increase this overwhelming domination. Thus our analysis quantitatively verifies the assertion by Nesvorný and Morbidelli (1998) that "the threebody mean motion resonances seem to be the main actors structuring the dynamics in the main asteroid belt".

8. We would like to point out that our results confirm the general concept of Molchanov $(1968,1969)$ on the omnipresence of resonances in the Solar system, however at a new level of understanding of this phenomenon.

\section{Acknowledgements}

The authors are thankful to Zoran Knežević and David Nesvorný for valuable remarks and comments.

This work was partially supported by the Russian Foundation for Basic Research (project \# 10-02-00383) and by the Programmes of Fundamental Research of the Russian Academy of Sciences "Fundamental Problems in Nonlinear Dynamics" and "Fundamental Problems of the Solar System Studies and Exploration". The computations were partially carried out at the St. Petersburg Branch of the Joint Supercomputer Centre of the Russian Academy of Sciences.

\section{References}

AstDyS website: http://hamilton.dm.unipi.it/cgi-bin/astdys/ (Maintained by A. Milani, Z. Knežević and their coworkers.)

Bretagnon P. An iterative method for the construction of a general planetary theory. Astron. Astrophys. 231, 561-570 (1990).

Chambers J. A hybrid symplectic integrator that permits close encounters between massive bodies. Mon. Not. R. Astron. Soc. 304, 793-799 (1999).

Chirikov B.V. A universal instability of many-dimensional oscillator systems. Phys. Reports 52, 263-379 (1979). 
Gallardo T. Atlas of the mean motion resonances in the Solar System. Icarus 184, 29-38 (2006).

Holman M.J., Murray N.W. Chaos in high order mean motion resonances in the outer asteroid belt. Astron. J. 112, 1278-1293 (1996).

Knežević Z., Tsiganis K., Varvoglis H. The dynamical portrait of the Veritas family region. In: Proceedings of "Asteroids, Comets, Meteors 2002" (ACM 2002). Ed. by B. Warmbein. Berlin: ESA, 2002. P. 335-338.

Milani A., Knežević Z. Asteroid proper elements and the dynamical structure of the asteroid main belt. Icarus 107, 219-254 (1994).

Molchanov A.M. The resonant structure of the Solar system. Icarus 8, 203-215 (1968).

Molchanov A.M. The reality of resonances in the Solar system. Icarus 11, 104110 (1969).

Morbidelli A. Modern Celestial Mechanics. Aspects of Solar System Dynamics. Padstow: Taylor and Francis, 2002.

Morbidelli A., Guzzo M. The Nekhoroshev theorem and the asteroid belt dynamical system. Celest. Mech. Dyn. Astron. 65, 107-136 (1997).

Morbidelli A., Nesvorný D. Numerous weak resonances drive asteroids toward terrestrial planets orbits. Icarus 139, 295-308 (1999).

Murray C., Dermott S. Solar System Dynamics. Cambridge: Cambridge University Press, 1999.

Murray N., Holman M. Diffusive chaos in the outer asteroid belt. Astron. J. 114, 1246-1259 (1997).

Murray N., Holman M., Potter M. On the origin of chaos in the asteroid belt. Astron. J. 116, 2583-2589 (1998).

Nesvorný D., Morbidelli A. Three-body mean motion resonances and the chaotic structure of the asteroid belt. Astron. J. 116, 3029-3037 (1998).

Nesvorný D., Morbidelli A. An analytic model of three-body mean motion resonances. Celest. Mech. Dyn. Astron. 71, 243-271 (1999).

OrbFit software and orbit9 integrator website: http://adams.dm.unipi.it/ orbmaint/orbfit/ (Maintained by OrbFit consortium.)

Shevchenko I.I. On the Lyapunov exponents of the asteroidal motion subject to resonances and encounters. In: "Near Earth Objects, our Celestial Neighbors: Opportunity and Risk" (Proc. IAU Symp. 236). Ed. by A. Milani, G.B. Valsecchi, and D. Vokrouhlicky. Cambridge: Cambridge Univ. Press, 2007. P. 15-29. 
Shevchenko I.I. The width of a chaotic layer. Phys. Letters A 372, 808-816 (2008).

Shevchenko I.I. Lyapunov and diffusion timescales in the solar neighborhood. Astrophys. J. 733, 39-46 (2011).

Tsiganis K., Knežević Z., Varvoglis H. Reconstructing the orbital history of the Veritas family. Icarus 186, 484-497 (2007). 


\section{Appendix A}

\section{Asteroids in pure three-body resonances with Jupiter and Saturn}

$13 \mathbf{3}$-1 (3): 954231102358511456174332249927767344934116442441 462184789648131599096288463667727917356974425760567820282703 $\begin{array}{llllllllll}91809 & 105839 & 112669 & 117065 & 117344 & 129360 & 138837 & 140653 & 141042 & 145390\end{array}$

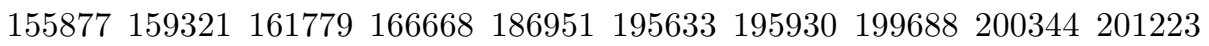
201875209429209437209473210159218079226525232332232494238387 239477240226

$\begin{array}{lllllllllllll}1 & 4 & \mathbf{- 1} & \mathbf{( 4 )}: & 9835 & 20596 & 31058 & 36595 & 37004 & 37611 & 41056 & 41640 & 50350\end{array}$ 510025506957488580026263266629674427102482600834718493887318 903689369997281107076119544122676126319130159147292158732160021

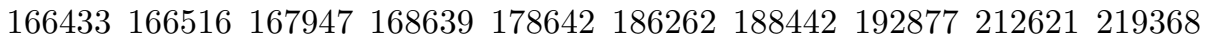
226015232815235341241349249070

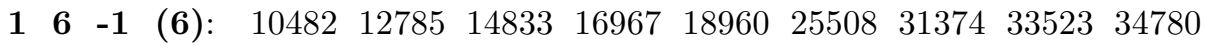

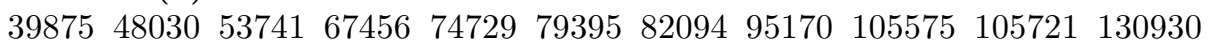
147022180268194048199297203374210376226238230570232392233526 245785

21 -1 (2): 114916703899267811105476167454229942241160

$\begin{array}{lllllllllllllll}2 & 2 & \mathbf{- 1} & \mathbf{( 3 )}: & 70 & 194 & 258 & 839 & 923 & 995 & 16233 & 28706 & 37158 & 48104 & 48110\end{array}$ 48127484184853251196620826732971407844379133793241117209122690 126001133792154671173752180242186145212288224957232199238618 243865

$\begin{array}{lllllllllllll}2 & 3 & \mathbf{- 1} & \text { (4): } & 7553 & 10368 & 39930 & 42942 & 48007 & 48028 & 48039 & 48105 & 48111\end{array}$ 481184812048121481334850948511694687051472106743168234189577 900159035490546112351113728115283119372122007125642129963130692

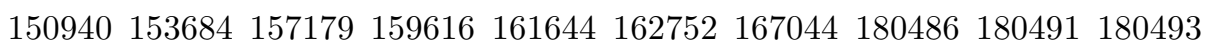
180494186531188874189427197995203427216047220119222186236300 239888243110244652248126

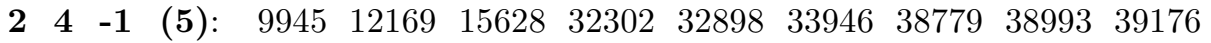
$4801448033481034810759305 \quad 752439085199504 \quad 120401 \quad 138965143630$ 168287180218211512217139218091239263

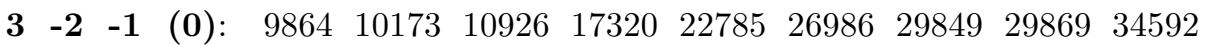
366714094041403453124791954497568835884758914629896477371551 734697357073573737987395275981765357875682905830118305583103 $83698 \quad 8397284448 \quad 84640900979420297229106316 \quad 112360112384112757$ 113085113337113402113904115445123903124012127061128255128304 


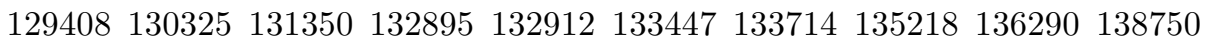
$\begin{array}{lllllllllll}140372 & 140655 & 143617 & 144889 & 152112 & 157195 & 158147 & 160008 & 160150 & 164493\end{array}$ $\begin{array}{lllllllllll}166768 & 166873 & 167567 & 169214 & 169298 & 170112 & 172023 & 173990 & 175484 & 182584\end{array}$ $\begin{array}{llllllllll}186114 & 189942 & 190125 & 195672 & 195779 & 195797 & 196016 & 196634 & 198642 & 199662\end{array}$ 201338201368202406203309203815204167207764209436209446209457

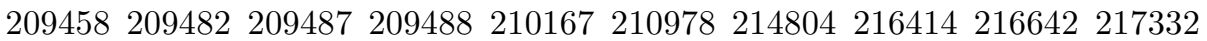
218433221358221408223303226565227722228301231654234751234855 234957235444235958240046243227243296246676247045248205249330

$\begin{array}{lllllllllllllll}3 & \mathbf{- 1} & \mathbf{- 1} & \mathbf{( 1 )}: & 564 & 947 & 1427 & 2042 & 2465 & 3534 & 4106 & 4426 & 5701 & 8068 & 9447\end{array}$ 130851335714169143681830219901201182069422146225622401124032 241902531425891278273119233071341043592637025411574186842743 443624445845486480384853950298509545408954405544295589356860 571615787959296596816016061974619806251863423649376554367013 670286734969300693757128171401714237142874215742257431476226 762387638778768794438184181883819278338883935877498789388110 $8817389354906479321293749954869699497230112181 \quad 114698 \quad 115643$ $\begin{array}{lllllllllll}117401 & 117668 & 118375 & 118835 & 120109 & 122501 & 122720 & 123213 & 123891 & 126305\end{array}$ $\begin{array}{lllllllllll}127119 & 127499 & 131131 & 135585 & 138054 & 138096 & 139828 & 139925 & 140586 & 140605\end{array}$ $\begin{array}{lllllllllll}140891 & 143738 & 146483 & 146507 & 147367 & 147370 & 148921 & 148949 & 148965 & 149535\end{array}$ $\begin{array}{lllllllllll}152057 & 156621 & 157792 & 159133 & 161375 & 162113 & 164762 & 170012 & 170043 & 170655\end{array}$ $\begin{array}{llllllllll}171450 & 172327 & 172703 & 172908 & 174418 & 177037 & 179311 & 180571 & 180686 & 181134\end{array}$ $\begin{array}{llllllllll}181576 & 181761 & 181872 & 182146 & 185159 & 185934 & 188312 & 192832 & 195353 & 196624\end{array}$

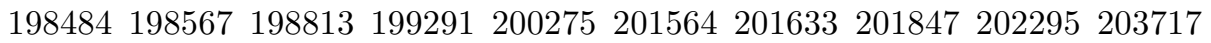
204124204800206078206622206654207269207302207661208908211245 212444213406217444217785217790218768218955219401220218220322 222347223648223656223845225299227701229534230119231697233422 234345235689239615241729242054244969245373245400247051

$\begin{array}{lllllllllllll}3 & 1 & \mathbf{- 1} & \mathbf{( 3 )}: & 1705 & 6581 & 10851 & 15431 & 22249 & 36579 & 41431 & 44783 & 48124\end{array}$ 500815212552586530905818559601646146616368674689966939369636

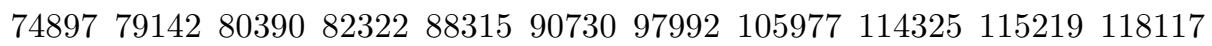
$\begin{array}{llllllllll}119371 & 125682 & 129748 & 129751 & 131646 & 134132 & 137237 & 139138 & 145918 & 153401\end{array}$

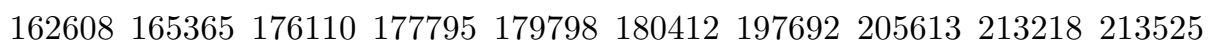
217478220043226224232412235665240796

$\begin{array}{lllllllllllll}3 & 3 & \mathbf{- 2} & \text { (4): } & 5413 & 10656 & 11653 & 21807 & 29946 & 41152 & 48515 & 48516 & 106548\end{array}$ 106707121883138820149695182619185797209431209440209464209469 209478209483209494223389

$\begin{array}{lllllllllllll}3 & 4 & \text {-2 } & \text { (5): } 2898432932 & 33778 & 36169 & 39340 & 42104 & 56894 & 113850 & 116063\end{array}$ 167477168326197436209435209439209443225316

34 -1 (6): 209454

4 -4 -1 (1): 52667107282129662157240163464198658 
$\begin{array}{lllllllllllll}4 & \mathbf{- 3} & \mathbf{- 1} & \mathbf{( 0 )}: & 3870 & 7456 & 7530 & 19700 & 21151 & 22085 & 22686 & 22904 & 23678\end{array}$ 239603371935565375413792739547399104270848130485355084257528 612666128461693668676846373206752817615076180785098128690130 90690907219393294135968779696699102105019105230112405113756 $\begin{array}{llllllllll}113933 & 119525 & 126653 & 126750 & 126821 & 132369 & 135793 & 138256 & 139761 & 144051\end{array}$ $\begin{array}{lllllllllll}148896 & 149892 & 150968 & 158721 & 162842 & 165625 & 168566 & 169085 & 171254 & 172708\end{array}$ $\begin{array}{llllllllll}178376 & 179009 & 182622 & 182698 & 186902 & 187380 & 195541 & 196456 & 205418 & 210213\end{array}$

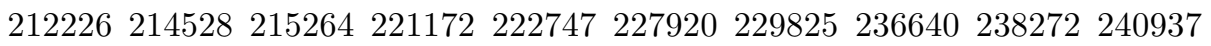
241763241766241991242342246382248308

$\begin{array}{lllllllllllllll}4 & \mathbf{- 2} & \mathbf{- 1} & \mathbf{( 1 )}: & 463 & 2348 & 2487 & 2640 & 2791 & 3048 & 3293 & 3699 & 3716 & 3733 & 3865\end{array}$ $46845156532357476167 \quad 6193694771337831804481668772977710009$ 100581009210218103711070111011112561206512097121611217712299 125101267412730129851300213024131271315913637137111424714823 157571616316451165251683716867171971736317729178691813018319 183911902919147196192041920658207682077520849215562213022150 223252232622797228292294723907241882571526295266652696027260 279482845129093294423074830799308743146631838318733196331971 328133380434047347073686836943381863869539720399583996340379 406244063540753410054151341697420614208142319426264265442666 430234349944184442244512845256459794725748101485134852650597 513995141251449515635158651628522525237452897530955386854082 542555435754374546985488055658560615661256617576685768957772 578125782258174586015912159413594895972160014602176041160790 609906111961650620986316863620643886456764685655406561565800 661426616566447670196728167938681686818368460694707014770224 703327034670376704097041370572714677197772081725967335273540 737097380273809738757409074589747837498175026750677525475317 753187536275495770347732078298785567894279038791267958379713 802378065880709807988101181017810708258584033840988419487288 883318858088743888178907189512895159012190503905159061590977 909889131992578926059286392879932379323894688951199527496372

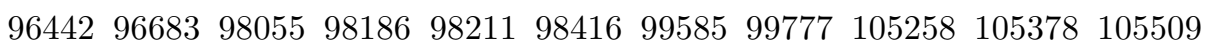
$\begin{array}{llllllllll}106090 & 106424 & 106862 & 106995 & 107111 & 112635 & 112731 & 113045 & 113416 & 113788\end{array}$ $\begin{array}{lllllllllll}114358 & 114381 & 114393 & 114414 & 115067 & 115148 & 115739 & 116691 & 117187 & 118096\end{array}$ $\begin{array}{llllllllll}119087 & 119128 & 119129 & 119252 & 119322 & 119327 & 120754 & 122038 & 122121 & 122144\end{array}$ $\begin{array}{lllllllllll}122171 & 122211 & 122987 & 124608 & 124893 & 124952 & 124961 & 125305 & 125404 & 125655\end{array}$ $\begin{array}{lllllllllll}125661 & 126059 & 126235 & 126356 & 127513 & 127711 & 127725 & 128307 & 128375 & 129225\end{array}$ $\begin{array}{lllllllllll}129343 & 129915 & 129940 & 130526 & 130622 & 130697 & 130774 & 130892 & 131004 & 131812\end{array}$

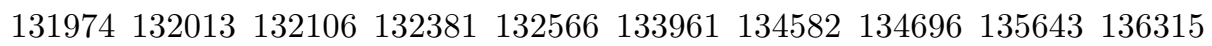
$\begin{array}{lllllllllll}136959 & 137506 & 137576 & 137736 & 137873 & 137940 & 137946 & 138116 & 138134 & 139266\end{array}$ $\begin{array}{llllllllll}139282 & 140628 & 141811 & 141814 & 142007 & 142021 & 142132 & 142238 & 142240 & 142267\end{array}$ $\begin{array}{lllllllllll}142288 & 142323 & 142338 & 142394 & 143253 & 145795 & 146052 & 146067 & 146075 & 146299\end{array}$ $\begin{array}{lllllllllll}146300 & 146327 & 146986 & 147613 & 147643 & 147886 & 147993 & 148006 & 148501 & 149381\end{array}$

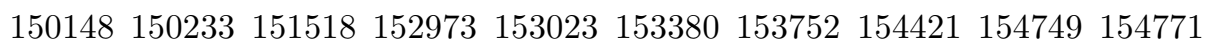


$\begin{array}{llllllllll}154903 & 155093 & 156074 & 156366 & 156875 & 156882 & 156905 & 158681 & 160447 & 160575\end{array}$ $\begin{array}{lllllllllll}161210 & 161308 & 162728 & 162772 & 163114 & 164841 & 164849 & 165283 & 165371 & 165490\end{array}$ $\begin{array}{lllllllllll}166087 & 166120 & 167081 & 167779 & 168144 & 168275 & 168802 & 168929 & 170267 & 170332\end{array}$ $\begin{array}{lllllllllll}170346 & 171028 & 171534 & 171560 & 173167 & 173174 & 173265 & 173320 & 173620 & 174191\end{array}$ $\begin{array}{llllllllllll}174824 & 175155 & 175400 & 175462 & 175491 & 175870 & 175885 & 175904 & 176707 & 177253\end{array}$ $\begin{array}{lllllllllll}177465 & 178603 & 178843 & 179629 & 180430 & 180580 & 181100 & 181104 & 181801 & 182290\end{array}$ $\begin{array}{lllllllllll}183153 & 183290 & 183786 & 183925 & 183934 & 183950 & 184459 & 185154 & 185710 & 186450\end{array}$ $\begin{array}{llllllllll}186857 & 187144 & 188383 & 189687 & 192429 & 192616 & 194342 & 194374 & 194425 & 194658\end{array}$ $\begin{array}{llllllllll}194663 & 194706 & 194804 & 194841 & 195074 & 195127 & 196258 & 196304 & 196390 & 196471\end{array}$ 198228198531198942200409200893200964201028201053201117202043 202257202262202504202533202949203615203902204399204470205002

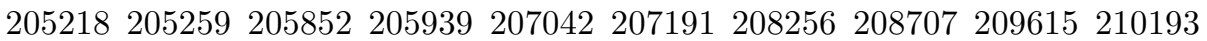

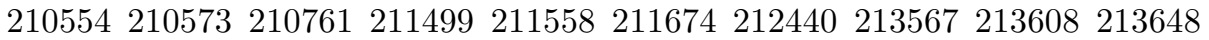

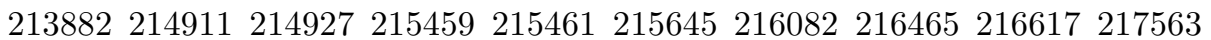

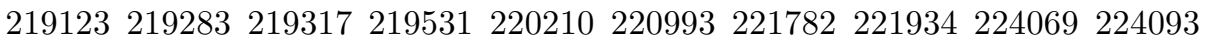

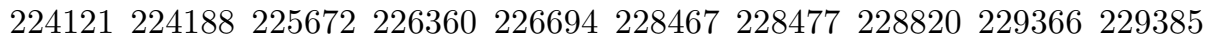

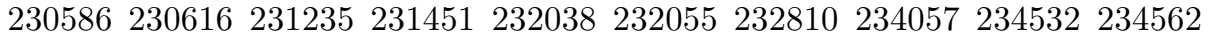

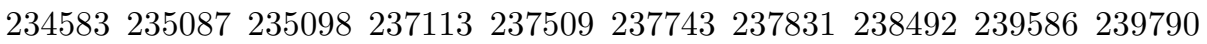
239898240346240581240869240898241625242784243623243785243827 244214244752244778246097246158246168247378247851248673

4 -1 -1 (2): 443217524401036420912294353032130916530116545567555 67699768769834298448106572116738121837124515127569129739137412

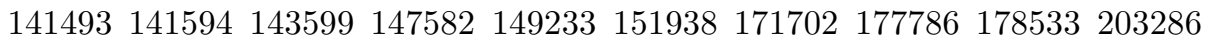
210206214109214530215602217517219155224790231602242502

41 -2 (3): 116793

41 -1 (4): 506628441141359166908209455

$\begin{array}{lllllllllllll}4 & 3 & \mathbf{- 2} & \mathbf{( 5 )}: & 37957 & 62712 & 81077 & 81561 & 115442 & 126605 & 170321 & 180727\end{array}$ 209153213348226790

$\begin{array}{lllllllllllll}\mathbf{5} & \mathbf{- 7} & \mathbf{- 1} & \mathbf{( 3 )}: & 19282 & 22198 & 48500 & 58123 & 58525 & 59375 & 82464 & 82946 & 83299\end{array}$ 84232846968757697536105807113343118397121291130281138591141285

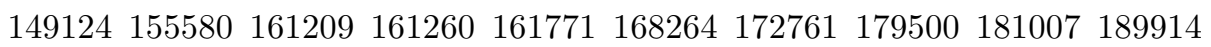
195767195867197461201308206843208354208477209442209444209463 209471209495212905217259224768237985238056242698

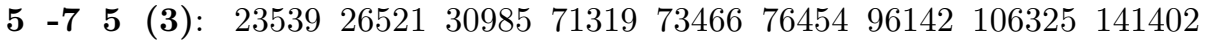
145415231035240779

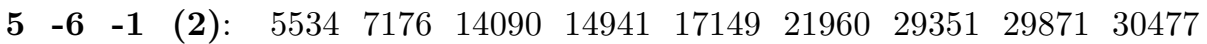
351533557539299411085065753321563106223862810680996872369897 705927095197743105868114380121278122957123365125842132699133224 139562146555147789150481153062164540170742176979177535178321 
195294201559221246224675230944237117243700249153

5 -6 5 (4): 43685219393

$\begin{array}{lllllllllllll}5 & \mathbf{- 4} & \mathbf{- 1} & \mathbf{( 0 )}: & 5277 & 9459 & 12814 & 13065 & 13757 & 16424 & 19419 & 22792 & 23520\end{array}$ 236572710027804296503032830679309593143932804334123571835991 364183642438097398104250543479445654518145842458884644547258 480105170851709528415295553791557055910659115606336145765886 661946626966295673856782968668695737175371909723277504679865 $803948085088936 \quad 89454924229252497837 \quad 111991 \quad 112301 \quad 114347 \quad 115038$ $\begin{array}{llllllllll}117889 & 118180 & 118353 & 121107 & 124291 & 124903 & 125165 & 125652 & 129793 & 131610\end{array}$ 131808137413137578137760139104139153142094145952147019153446

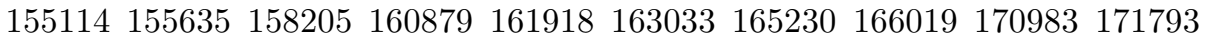

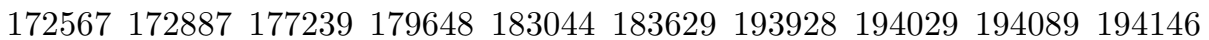
194297201491202894205754207455208634211549212425215432216611 217155217399221620222939226260229061230577232383236196238076 245780

5 -3 -1 (1): 78822999843935106173107454207395220455221522

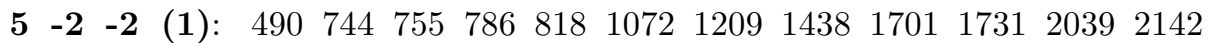
$21642250249225872666273127572863 \quad 3460415268309026916311973$ 127621291214360169681716517216182022122321547215762209725681 295192988129931310063174832416332253399734070351833566038250 385074071845587460954765247949519265225752693533515568257870 591606255164977660296699068861699777003771628733367679379488 818918199988003901779026390732907499419895638975519773397763 977729914599254106033106113114278115826121308121556121789123740 $124027124040127308130258132983133482 \quad 134109 \quad 138716141162141215$ $\begin{array}{llllllllll}141236 & 141351 & 143648 & 144814 & 149972 & 153256 & 153259 & 154145 & 155322 & 157670\end{array}$ $\begin{array}{llllllllll}159244 & 159526 & 159815 & 159972 & 168466 & 173044 & 173077 & 175803 & 177910 & 179478\end{array}$ $\begin{array}{llllllllll}181086 & 181160 & 183563 & 184199 & 189504 & 189885 & 195674 & 196076 & 196130 & 197452\end{array}$ 198665198785201774206892209499210746214499214566214732215565

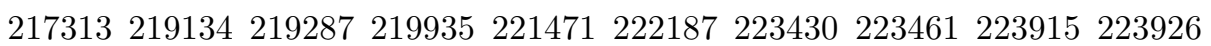

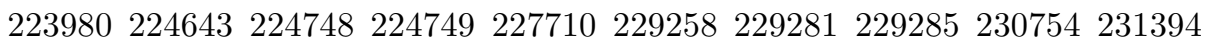
232598235502236088237605239525239696240726240783242592242713 242924244065248258248482248939

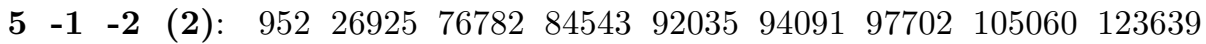
129074145233163463206169209432217709218420222105222396223884 229592237971242560

5 -1 -1 (3): 97741209474

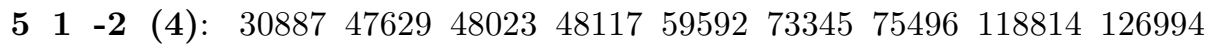
148536149752184749 


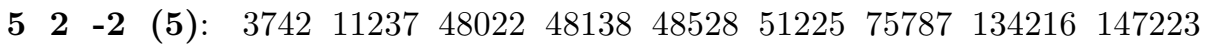
150902166364173403180497190240192315221121227919

53 -3 (5): 411885191952365

$\begin{array}{lllllllllllll}5 & 3 & \mathbf{- 2} & \text { (6): } & 28572 & 48012 & 48035 & 63365 & 69144 & 70259 & 77353 & 107672 & 118218\end{array}$ $\begin{array}{lllllllllll}147173 & 153032 & 173280 & 177352 & 180405 & 180485 & 180487 & 180488 & 180490 & 180495\end{array}$ 180496180499181592195111207078208062213634217526218932220275 220992

$\begin{array}{lllllllllllll}6 & \mathbf{- 7} & \mathbf{- 1} & \mathbf{( 2 )}: & 9905 & 36976 & 45278 & 48016 & 48137 & 53029 & 56026 & 61235 & 64335\end{array}$ 65955730939486399656128688132181134246142870145801154396158662

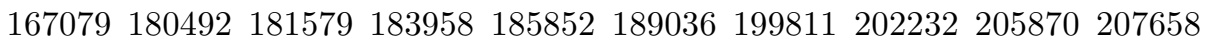
213096214864217403224231232466

6 -7 5 (4): 21416122051180505224163228870

54 -3 (6): 204141

$\begin{array}{lllllllllllll}6 & \mathbf{6} & \mathbf{- 1} & \mathbf{( 1 )}: & 5684 & 60723 & 61210 & 77084 & 77140 & 78925 & 86835 & 92462 & 129754\end{array}$

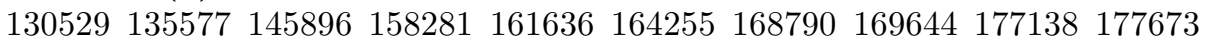
192546199074216654234556

6 -4 -1 (1): 4800197759141744157079217839

$\begin{array}{llllllllllll}6 & \mathbf{- 3} & \mathbf{- 2} & \text { (1): } & 13797 & 19987 & 107393 & 122771 & 132844 & 204025 & 209370 & 237930\end{array}$ 244227246410

6 -3 -1 (2): 76800

6 -1 -2 (3): 342611792208619134798826142623148819170636196499200520

$\begin{array}{llllllllllll}6 & \mathbf{1} & \mathbf{- 3} & \mathbf{( 4 )}: & 57861 & 69553 & 76017 & 78776 & 116376 & 159801 & 168157 & 171677\end{array}$ 184224202322209447209449209451209453209462209480209484219975 232662238386240451

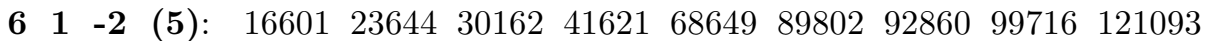
$\begin{array}{llllllllll}132149 & 134702 & 139246 & 142066 & 150091 & 155432 & 163292 & 169468 & 188240 & 194745\end{array}$ 197727197853211543215096220219228253230149241162

$\begin{array}{lllllllllllll}6 & \mathbf{2} & \mathbf{- 3} & \mathbf{( 5 )}) & 4600 & 41401 & 51861 & 121579 & 159818 & 184675 & 209434 & 209467\end{array}$ 244168

$\begin{array}{llllllllllll}\mathbf{7} & \mathbf{- 7} & \mathbf{- 2} & \mathbf{( 2 )}: & 15290 & 15731 & 23485 & 47732 & 113231 & 170857 & 177020 & 177095\end{array}$ 181071209450212665216400236505248433 


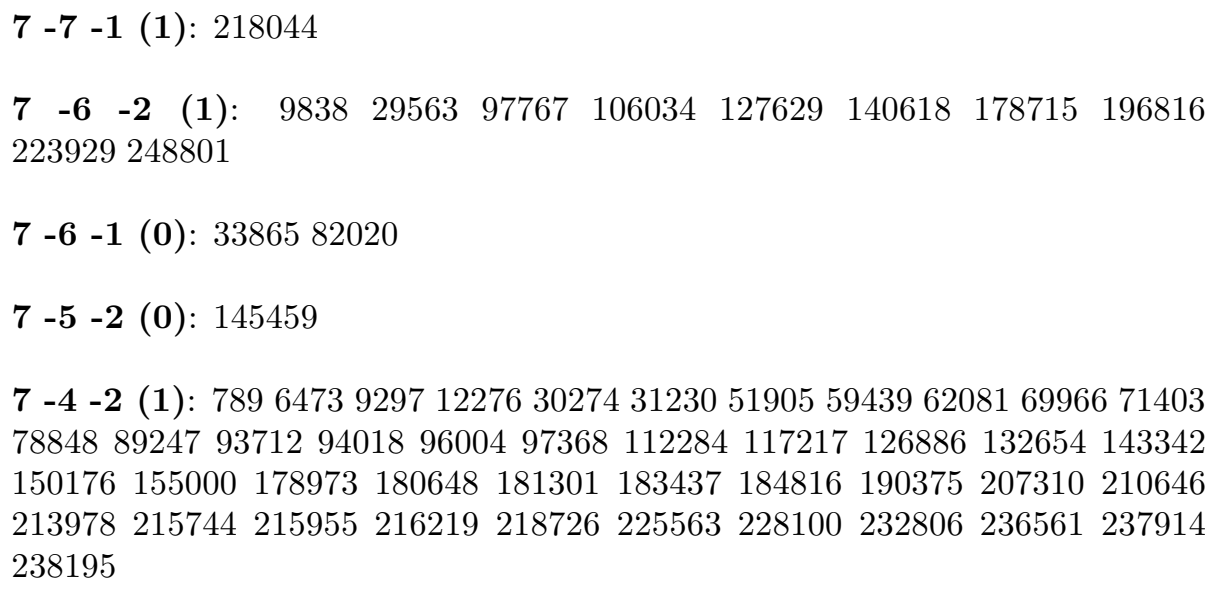

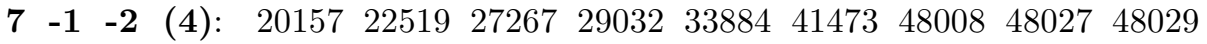
5017751198537665738762411729097915779676887429506899518125118 125567130635132085132091147946154375158303159034163148170184 174793175160175831213528214159218114240121245749

71 -3 (5): 516645793565732116950190207223715

$7 \mathbf{1}$-2 (6): 480204803168793131749174692

72 -3 (6): 633046688079094145551219356237227237964240175

7 3 -4 (6): 120291

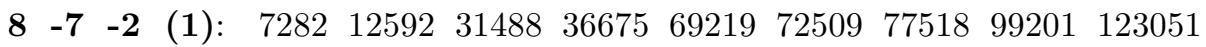
150021157386161417166474181601182133185382186607196547204898 206018219402232499236599244538

8 -4 -3 (1): 104685125056857113931134378173978209448209485

8 -3 -3 (2): 105866 
8 -3 -2 (3): 979121634105959106040136430181378200353225201

8 -2 -3 (3): 151028203738

8 -1 -3 (4): 1969841253160914185903

8 -1 -2 (5): 181412

81 -4 (5): 221458

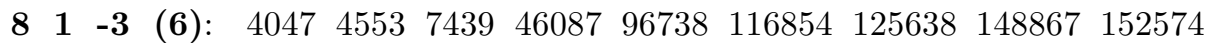
219335 


\section{Appendix B}

\section{Asteroids in pure two-body resonances with Jupiter}

1/1 (0): 58861762465988491111431172120814041437158316471749 186718691870187118732146214822072223235723632456267427592797 289328952920306332403317339134513540354835643596370837093793 379440074035405740604063406840864138448945014707470847094715 472247544791479248054827482848294832483348344835483648674902 501250235025502750285041511951205123512651305144520952335244 525452575258525952645283528452855436547655115637563856485652 590760026443654569976998711971527214735275437641781580608125 824183179023903091429430943195909694971297139790979998079817 9818982898579907102471066410989110891125111252112731127511351 113951139611397114291148711488115091155211554116631166811869

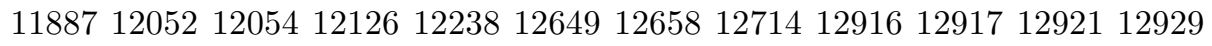
129721297312974130601306213181131821318313184131851322913323 133531336213366133721337913383133851338713402134631347513650 136941378013782137901386214235142681451814690147071479114792 150331509415398154361544015442155021552115527155291553515536 $15651156631607016099161521642816667169561697417171 \quad 1717217314$

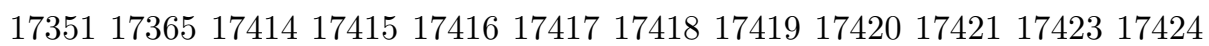
174421749217874180371804618054180581806018062180631807118137 182281826318268182781828118282189401897119018190201972519844 199132014420424204282071620720207292073820739209472095220961 212712128421370213712137221595215992160121602219002200822009 220102201222014220352204122042220522205422055220562205922149 221802220322227224042250322808230752311423118231192312623135 231442315223269232852335523382233832346323480235492362223624 236942370623709237102394723958239632396823970239872401824022 253442534725883258952591025911259372593826057264862651026601 267052895829196293142960329976299773002030102304983049930504 305053050630510306983070430705307083079130792307933080630807 309423103731342318063181931820318213183532339323563237032396 323973243432435324373244032451324613246432467324713247532478 324803248232496324983249932501325133261532720327263279432811 338223429834553346423468434746347853499335272352763527735363 356723567336259362653626736268362693627036271364253662436922 372973729937300373013751937572376853771037714377153771637732 377893779038050380513805238257385743858538592385943859638597 385983859938600386063860738609386103861138614386153861738619 386213922939264392703927539278392803928439285392863928839289 392923929339369394633947439691396923969339793397943979539797 397984023740262412684134041350413534135541359413794141741426 414274203642114421464216842176421794218242187422014223042277 423674255442555432124343643627437064582246676479554796247963 
479644796747969484385133951340513445134551346513475134851350 513515135451357513595136051362513645136551378519105193551958 519625196951984519945227352275522785251152567526455341853419 534365344953469534775458154582546145462654632546345463854643 546455464654649546525465354655546565467254678546805468955060 552675541955441554575546055474554965556355568555715557455676 556785570155702563555695156962569685697657013576265771457904 579105791557920580085808458096581535836658473584755847858479 584805893159049593556031360322603286038360399604016042161610 618966211462201624266269262714631756319363195632026321063234 632396324163257632596326563269632726327363278632796328463286 632876329063291632926329464270643266500065097651096511165134 651506517465179651946520565206652096521065211652236522465225 652276523265240652436524565250652576528165583655906581167548 681126844468725687666878869437736377364173677737957680476809 768127681976820768247682676830768347683576836768377683876840 768577686777891778947789777902779147791679444801198030280638 820558397583978839798398083983847098822588227882298824088241 882458877589829898368984189844898528985889871898728987889886 898988991389918899228992489927899348993589938899409033790380 905699629597893979739803798116981399836199306993089930999311 993239932899368994649994399950100475100619100624101405101466

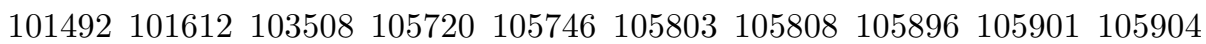
$\begin{array}{lllllllllll}106001 & 106060 & 106091 & 106160 & 107004 & 107134 & 107178 & 111932 & 114141 & 114208\end{array}$ $\begin{array}{llllllllll}114345 & 114694 & 114710 & 116134 & 116238 & 116439 & 116567 & 116901 & 116930 & 116954\end{array}$ $\begin{array}{lllllllllll}116969 & 116970 & 117389 & 117395 & 117404 & 117423 & 117446 & 117447 & 117832 & 117851\end{array}$ $\begin{array}{lllllllllll}118815 & 119528 & 120453 & 120454 & 122391 & 122460 & 122581 & 122592 & 122733 & 122860\end{array}$ $\begin{array}{lllllllllll}122862 & 124695 & 124696 & 124729 & 124985 & 125045 & 125048 & 125059 & 125062 & 125106\end{array}$ $\begin{array}{lllllllllll}125159 & 127532 & 127534 & 127846 & 128299 & 128301 & 128383 & 129130 & 129133 & 129134\end{array}$ $\begin{array}{llllllllll}129137 & 129140 & 129142 & 129144 & 129145 & 129147 & 129153 & 129207 & 129583 & 129602\end{array}$ $\begin{array}{lllllllllll}130190 & 130588 & 130592 & 130687 & 131447 & 131460 & 131539 & 131546 & 131581 & 131635\end{array}$ $\begin{array}{llllllllll}133566 & 133853 & 133862 & 134251 & 134269 & 134329 & 134419 & 134720 & 134749 & 134957\end{array}$ $\begin{array}{llllllllll}134965 & 135540 & 135547 & 135593 & 135594 & 136557 & 137879 & 137954 & 138031 & 139009\end{array}$ $\begin{array}{llllllllll}141577 & 141584 & 150872 & 150876 & 151883 & 151884 & 152297 & 152517 & 152519 & 153083\end{array}$ $\begin{array}{lllllllllll}153107 & 153122 & 153141 & 153155 & 153464 & 153500 & 153708 & 153755 & 153757 & 153758\end{array}$ $\begin{array}{lllllllllll}154417 & 154632 & 154989 & 154990 & 154992 & 155326 & 155327 & 155332 & 155337 & 155415\end{array}$ $\begin{array}{lllllllllll}155427 & 155786 & 155789 & 156098 & 156123 & 156125 & 156185 & 156188 & 156210 & 156237\end{array}$ $\begin{array}{lllllllllll}156250 & 156252 & 156293 & 156294 & 156730 & 157468 & 157469 & 157470 & 157471 & 157740\end{array}$ $\begin{array}{llllllllllll}157741 & 158231 & 158333 & 158336 & 158601 & 159162 & 159163 & 159340 & 159342 & 159378\end{array}$ $\begin{array}{lllllllllll}159633 & 159658 & 160135 & 160140 & 160164 & 160465 & 160527 & 160528 & 160533 & 160534\end{array}$ $\begin{array}{lllllllllll}160661 & 160679 & 160856 & 161003 & 161020 & 161024 & 161027 & 161044 & 161484 & 161489\end{array}$ $\begin{array}{lllllllllll}161701 & 161717 & 162352 & 162380 & 162388 & 162396 & 162805 & 162811 & 162822 & 162851\end{array}$ $\begin{array}{lllllllllll}162861 & 163102 & 163135 & 163155 & 163189 & 163196 & 163208 & 163216 & 163238 & 163256\end{array}$ $\begin{array}{llllllllll}163263 & 163731 & 164208 & 164210 & 164212 & 164585 & 165531 & 165551 & 165569 & 166115\end{array}$ $\begin{array}{lllllllllll}166123 & 166136 & 166148 & 166211 & 166230 & 166285 & 166351 & 167686 & 168033 & 168280\end{array}$

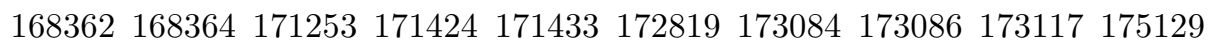


$\begin{array}{llllllllll}175471 & 177655 & 178268 & 178291 & 178387 & 179133 & 179190 & 179217 & 179233 & 179244\end{array}$ $\begin{array}{lllllllllll}179902 & 180265 & 180274 & 181278 & 181279 & 181648 & 181665 & 181751 & 181773 & 182121\end{array}$ $\begin{array}{lllllllllll}182161 & 182163 & 182176 & 182178 & 182445 & 182487 & 182506 & 182516 & 182522 & 182548\end{array}$ $\begin{array}{lllllllllll}182625 & 182627 & 182647 & 182666 & 182669 & 182675 & 182746 & 183309 & 183358 & 184274\end{array}$ $\begin{array}{llllllllllll}184276 & 184280 & 184284 & 184306 & 184829 & 184937 & 184975 & 184976 & 184977 & 184978\end{array}$ $\begin{array}{llllllllllll}184979 & 184980 & 184981 & 184982 & 184983 & 184984 & 184986 & 184988 & 185485 & 185486\end{array}$ $\begin{array}{lllllllllll}185487 & 185489 & 185490 & 185492 & 185666 & 185693 & 185906 & 185919 & 186106 & 186125\end{array}$ $\begin{array}{lllllllllll}186128 & 186138 & 186272 & 186544 & 187018 & 187019 & 187021 & 187024 & 187436 & 187456\end{array}$ $\begin{array}{lllllllllll}187463 & 187469 & 187470 & 187471 & 187472 & 187473 & 187474 & 187475 & 187476 & 187478\end{array}$ $\begin{array}{lllllllllll}187479 & 187607 & 187655 & 187657 & 187659 & 187692 & 187724 & 187755 & 188006 & 188020\end{array}$ $\begin{array}{llllllllllll}188026 & 188053 & 188060 & 188234 & 188247 & 188257 & 188574 & 188835 & 188836 & 188837\end{array}$ $\begin{array}{llllllllllll}188841 & 188842 & 188843 & 188844 & 188845 & 188846 & 188847 & 188942 & 188952 & 188965\end{array}$ $\begin{array}{lllllllllll}188966 & 188976 & 188986 & 189004 & 189214 & 189215 & 189310 & 189377 & 189386 & 189493\end{array}$ $\begin{array}{lllllllllll}189616 & 189772 & 189775 & 189859 & 190005 & 190229 & 190264 & 190267 & 190268 & 190294\end{array}$ $\begin{array}{llllllllll}190301 & 190309 & 190311 & 190351 & 190352 & 190353 & 190442 & 190446 & 192215 & 192217\end{array}$ $\begin{array}{llllllllll}192218 & 192220 & 192221 & 192222 & 192223 & 192224 & 192262 & 192268 & 192343 & 192345\end{array}$ $\begin{array}{llllllllll}192386 & 192388 & 192389 & 192390 & 192393 & 192423 & 192442 & 192448 & 192929 & 192938\end{array}$ $\begin{array}{lllllllllll}192942 & 192964 & 193535 & 193570 & 193592 & 193602 & 193641 & 193670 & 195041 & 195084\end{array}$ $\begin{array}{llllllllll}195104 & 195117 & 195126 & 195152 & 195153 & 195167 & 195188 & 195217 & 195218 & 195230\end{array}$ $\begin{array}{llllllllll}195245 & 195258 & 195269 & 195273 & 195284 & 195286 & 195287 & 195308 & 195309 & 195312\end{array}$ $\begin{array}{llllllllll}195315 & 195318 & 195324 & 195337 & 195351 & 195490 & 195505 & 196316 & 196318 & 196364\end{array}$ $\begin{array}{llllllllll}196408 & 196440 & 196488 & 197563 & 197586 & 197593 & 197619 & 197620 & 197624 & 197628\end{array}$

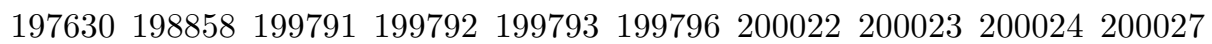
200028200029200032200035200036200037200046200051200057200544

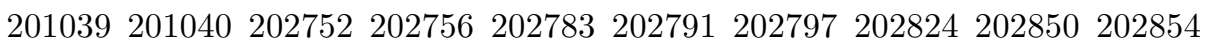
202855203210203865203891204619204847204911204927205380207749 210237211992212694213180213347213351213360213394214093214376 214506214508214511214514215020215107215110215199215243215319

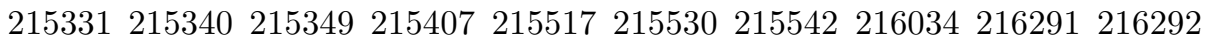
216293216307216409216419216421216423216462216847216876216877

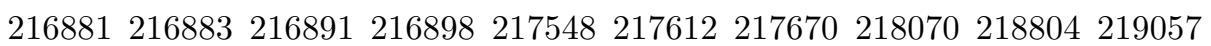
219070219139219140219141219466219834219835219837219857219866 219881219890219892219896219897219902219905219907219954219958 220312220318220324220333220335220336220342220351220355220356

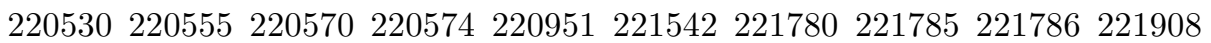
221909221910221911221912221913221914221915221917221995222049

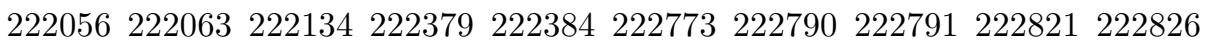

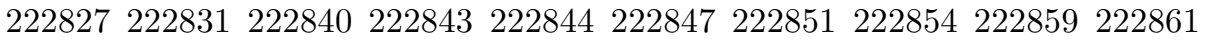

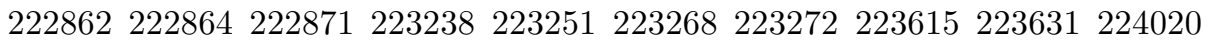

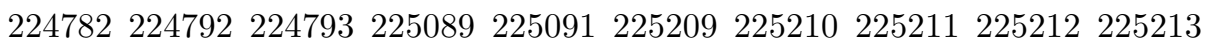

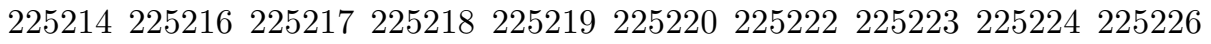

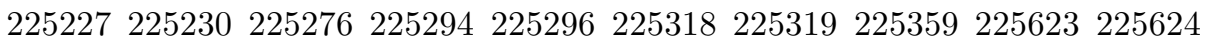

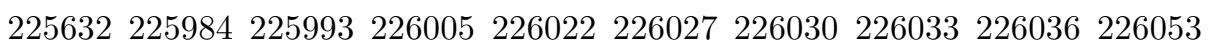

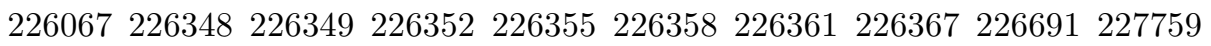
228005228006228007228008228009228010228071228077228084228085 228088228089228090228095228096228097228098228099228101228102 


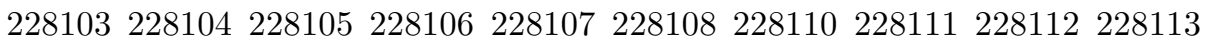
$\begin{array}{llllllllll}228114 & 228115 & 228116 & 228117 & 228118 & 228119 & 228120 & 228121 & 228122 & 228123\end{array}$ $\begin{array}{llllllllll}228124 & 228125 & 228140 & 228150 & 228155 & 228162 & 228239 & 229760 & 229761 & 229808\end{array}$

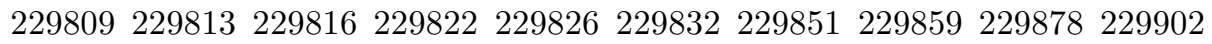
229998230339231464231493231516231572231610231612231615231620 $\begin{array}{llllllllll}231621 & 231623 & 231636 & 231664 & 231692 & 233578 & 233600 & 233683 & 233773 & 233790\end{array}$ $\begin{array}{llllllllll}233827 & 233915 & 233925 & 236878 & 236974 & 237035 & 237332 & 237338 & 237710 & 238623\end{array}$ 239521239903240914241099241573241576241581241582241589241643

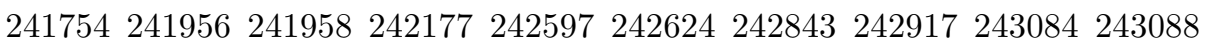

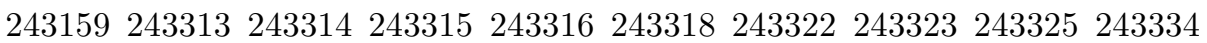
243453243460243479243484243490243494243496243501243504243509

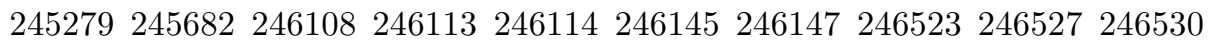
246533246534246546246550246560246817246831246833246891247019 247102247297247315247323247341247351247409247421247437247653 $\begin{array}{llllllllll}247946 & 247967 & 247969 & 248048 & 248183 & 248186 & 248189 & 248238 & 248550 & 248575\end{array}$ 248603248670248976248978248979248985248986248990248991248996 249005249032249180249212249225249236249237249241249247249256 249258249465249476249481249486

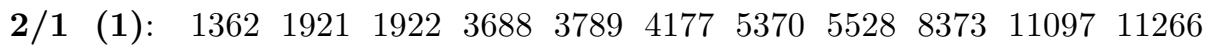
115731166513963148711688218888227402357726553283682845929524 312493129334901359893614037357375283799138406385363858438984 390183930941262422024551145796461684620447547510705126751537 527005519255222555225632657838626796276065297655416617468706 687387166171694733967397273995766947786977911781547880178814 792827948279500820098390483943873628737589175899089118291875 9594297406976539898899686100100102419102915104742107690113723 $\begin{array}{llllllllll}114804 & 115916 & 116236 & 116559 & 117645 & 120907 & 120976 & 121675 & 121812 & 121824\end{array}$ $\begin{array}{llllllllll}124086 & 126119 & 127209 & 128313 & 128442 & 130295 & 130380 & 130385 & 131443 & 135454\end{array}$ $\begin{array}{llllllllll}135496 & 136807 & 138699 & 138739 & 138816 & 138854 & 140348 & 140956 & 141109 & 141127\end{array}$ $\begin{array}{llllllllll}141370 & 141394 & 143411 & 144443 & 145545 & 146258 & 146951 & 148125 & 149018 & 149613\end{array}$ $\begin{array}{llllllllll}152835 & 154621 & 154835 & 154904 & 154906 & 155030 & 155121 & 155874 & 155890 & 158152\end{array}$ $\begin{array}{lllllllllll}159367 & 160013 & 161218 & 163660 & 165913 & 166564 & 166783 & 166912 & 167683 & 168337\end{array}$ $\begin{array}{llllllllll}169281 & 170869 & 171204 & 171414 & 172058 & 174006 & 174016 & 175976 & 178104 & 181067\end{array}$ $\begin{array}{lllllllllll}182888 & 182931 & 185140 & 185483 & 185809 & 186358 & 186982 & 187001 & 187648 & 188795\end{array}$ $\begin{array}{lllllllllll}188945 & 189375 & 190213 & 192606 & 193810 & 194142 & 196081 & 196114 & 196195 & 197406\end{array}$

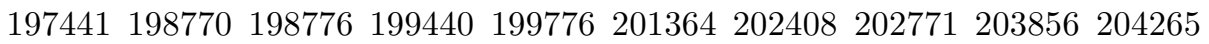
205487206286206297206298208517209024209450209489209496209499

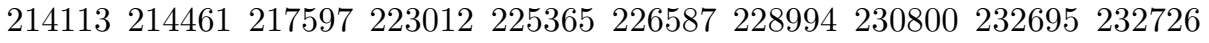
232745233635234059235455235546235547236934237665243192244606 245143245382246128

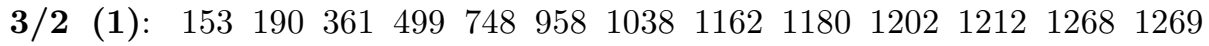
134514391512152915781746174817541877190219111941206723122483 262427602959313432023254329034153514355735613571357736553694 384339233990423042554317444644954757536854395603566157115928 
612462376984702771747284808681308376855085518743891389159661 98291006310296103311060810610106321117511249112741138811410 115421173911951120061230712896129201303513317133811350413897 141951456914669148451506815231152781537315376154261550515540 155451561515638156711578316232168431691516927169701721217305 174281786718036190341975220038206282063020640210472112821804 219302205822070226472269923174231862330123405258002586926761 269292756128918294332959129944299733043530764310203109731284 313383181732395324553246032724337533491935016356303618236274 369413715537452375783759038046382923847038553385793861338684 387013870938830392663928239294393013938239405394153942740227 402384024641278412834136541419414884216742190422374381843940 445494573945850458624630246629479074852951178512845129851349 518385187451885518885193051983527025451454599546285463054631 546445465755196554395549855505569825698556996570275775958095 581885827958353590505907959112602326031860381603986104262145 622416224462408624896282062959631846324963293634886349164390 647396482365236652446537465389658216585965989661876622767203 672466734067368682476828768374684026893369302694176956673418 734367345573457734587347573654737697388674051740547675076805 768107681176822768317773477820778847789277893778957790377905 779107813378159784707847778809788157909679097791907943979515 797248201182023820418204382044837228380183804838678387783903 839168401184103877048781187956882308823788246882538992890456 905029073791273913049227292281922839228492287923269234494266 942759429995952959579608696180966099770098002992519927699850 998629987799982100133100229100231101153101965103537104102104943 $105935111928111995112432112442112553 \quad 112586112694112822113224$ $\begin{array}{lllllllllll}113403 & 113415 & 114513 & 114749 & 114830 & 114837 & 114937 & 114954 & 114997 & 115088\end{array}$ $\begin{array}{llllllllll}115097 & 115170 & 115315 & 115380 & 115440 & 115463 & 116280 & 116294 & 116489 & 116512\end{array}$ $\begin{array}{llllllllll}116784 & 117108 & 117113 & 117131 & 117200 & 117288 & 117667 & 117993 & 118177 & 118520\end{array}$ $119904119918119922119935119942119944119945119946 \quad 119950120175$ $\begin{array}{lllllllllll}120179 & 120287 & 120329 & 120336 & 120363 & 120618 & 120662 & 120761 & 120962 & 121005\end{array}$ $\begin{array}{llllllllll}121045 & 121074 & 124100 & 124174 & 124269 & 124791 & 125130 & 127426 & 127442 & 127448\end{array}$ $\begin{array}{llllllllll}127449 & 127469 & 127519 & 128059 & 128121 & 128126 & 128175 & 128198 & 128203 & 128209\end{array}$ $\begin{array}{llllllllll}128235 & 128254 & 128295 & 128420 & 128632 & 128858 & 129002 & 129007 & 129079 & 129241\end{array}$ $\begin{array}{llllllllll}129634 & 130453 & 130609 & 131421 & 131468 & 131481 & 131502 & 132868 & 132904 & 133324\end{array}$ $\begin{array}{llllllllll}133559 & 133614 & 134193 & 134233 & 134429 & 134562 & 134606 & 134642 & 134652 & 134690\end{array}$ $\begin{array}{llllllllll}134909 & 136822 & 136835 & 136935 & 137011 & 137014 & 137015 & 137312 & 137349 & 137816\end{array}$ $\begin{array}{lllllllllll}138388 & 139799 & 141503 & 141518 & 141557 & 141701 & 142470 & 143621 & 143658 & 144851\end{array}$ $\begin{array}{lllllllllll}144866 & 144894 & 145368 & 145373 & 145396 & 145397 & 145417 & 145421 & 145422 & 145426\end{array}$ $\begin{array}{lllllllllll}145437 & 145443 & 145473 & 145478 & 145718 & 145767 & 145806 & 145833 & 145841 & 145843\end{array}$ $\begin{array}{llllllllll}145960 & 146014 & 146035 & 146961 & 147836 & 147846 & 147865 & 148060 & 148227 & 148234\end{array}$ $\begin{array}{llllllllll}149774 & 150033 & 150416 & 152094 & 152122 & 152132 & 152328 & 152373 & 152900 & 153357\end{array}$ $\begin{array}{lllllllllll}153386 & 154262 & 154263 & 154264 & 154603 & 154629 & 154932 & 154960 & 154971 & 155125\end{array}$ $155954155959156044156085156717157014 \quad 157025157076 \quad 157375157710$ 


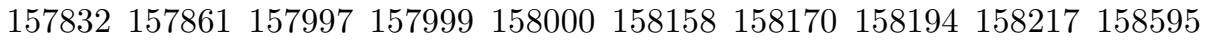
$\begin{array}{lllllllllll}158811 & 159339 & 159550 & 159937 & 160005 & 160234 & 160826 & 161334 & 162227 & 162232\end{array}$ $\begin{array}{lllllllllll}162256 & 162280 & 162284 & 162314 & 162705 & 162777 & 162796 & 164843 & 164844 & 164858\end{array}$ $\begin{array}{lllllllllll}164903 & 165044 & 165188 & 165306 & 165558 & 168517 & 168520 & 168525 & 168530 & 168545\end{array}$ $\begin{array}{lllllllllll}168550 & 168557 & 169456 & 169508 & 169509 & 171258 & 171420 & 171465 & 171513 & 171562\end{array}$ $\begin{array}{lllllllllll}171585 & 171600 & 172818 & 172965 & 172966 & 173012 & 173071 & 173083 & 173108 & 173351\end{array}$ $\begin{array}{llllllllllll}173543 & 173643 & 174074 & 174077 & 174089 & 174114 & 174121 & 175115 & 175117 & 175350\end{array}$ $\begin{array}{lllllllllll}175351 & 175355 & 175371 & 175372 & 175460 & 175620 & 175936 & 176158 & 176621 & 177640\end{array}$ $\begin{array}{lllllllllll}177898 & 177940 & 177941 & 177943 & 178295 & 178299 & 181254 & 181256 & 181275 & 181618\end{array}$ $\begin{array}{lllllllllll}181633 & 181647 & 181675 & 181778 & 181940 & 182062 & 182069 & 182094 & 182204 & 182239\end{array}$ $\begin{array}{lllllllllll}183113 & 183579 & 184225 & 184241 & 184256 & 184780 & 184857 & 184930 & 184952 & 184955\end{array}$ $\begin{array}{lllllllllll}184961 & 184963 & 185599 & 185640 & 185861 & 186448 & 186646 & 186649 & 187003 & 187011\end{array}$ $\begin{array}{lllllllllll}187012 & 187017 & 187303 & 187378 & 187423 & 187477 & 187646 & 188188 & 188341 & 188342\end{array}$ $\begin{array}{lllllllllll}188351 & 188830 & 188831 & 189114 & 189702 & 189714 & 189977 & 192203 & 193241 & 193248\end{array}$ $\begin{array}{llllllllll}193268 & 193291 & 193293 & 193306 & 193311 & 193323 & 193332 & 193350 & 193354 & 193398\end{array}$ $\begin{array}{llllllllll}193449 & 193501 & 194207 & 194384 & 194501 & 194512 & 194631 & 194724 & 194741 & 194742\end{array}$ $\begin{array}{llllllllllll}194743 & 194860 & 194927 & 194989 & 195204 & 197478 & 197528 & 197537 & 197554 & 197558\end{array}$ 198722199715200040200043200417200441200445200465200902201937 202314202551202965202975202982202992203128203156203157203159 204622204803204851204856204892205070205104205134207369207379

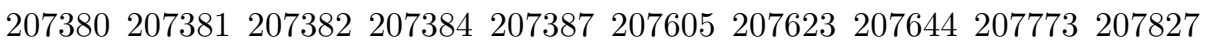
207853208015208290208695209031209507209512209919209920210049 210087210149210184210187210340212337212344212345212369212490 212690212928214472214483214910215089215377216408216411216613 $\begin{array}{llllllllll}216615 & 216771 & 217023 & 217024 & 217032 & 217039 & 217196 & 217208 & 217604 & 217844\end{array}$

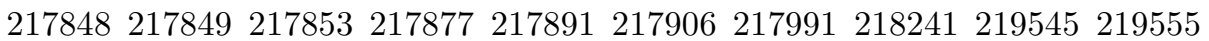
$\begin{array}{llllllllll}220461 & 220910 & 221778 & 221982 & 222002 & 222427 & 222450 & 222454 & 222473 & 222488\end{array}$

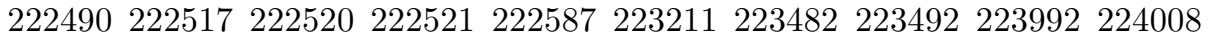

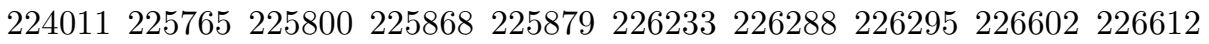

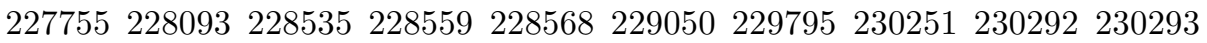
230294230309230334231400231745231749231872231909231913232088

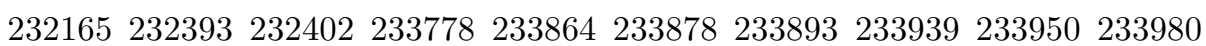

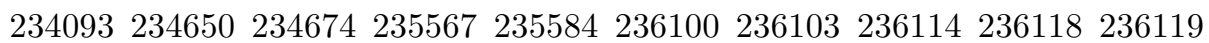

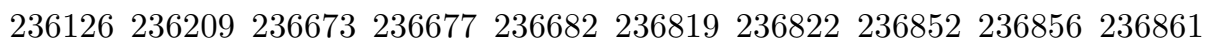

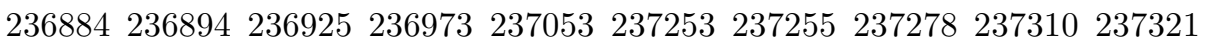
$\begin{array}{llllllllll}237323 & 237849 & 237858 & 237861 & 238114 & 238154 & 238560 & 238804 & 238818 & 238819\end{array}$

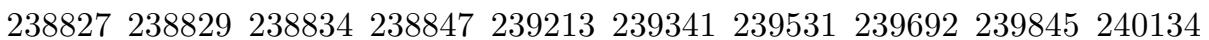
241093241376241528241563241994242008242016242148242149242159 242178242290242305242309242311242321242370242451242656242682

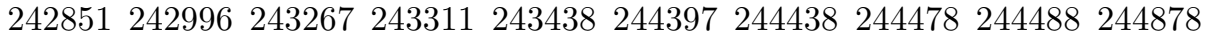
245007245093245216245578246178246496246497246578246726246786

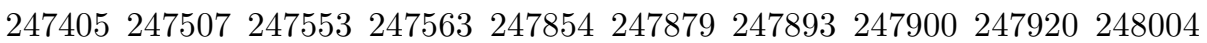
248430248945249017249060249182249246249266249275249284249302 249324249391249416

3/1 (2): $8887 \quad 6318 \quad 48012 \quad 48016 \quad 48017 \quad 48022 \quad 48024 \quad 48032 \quad 48034 \quad 48035$ 
4804048138481408692492664131702144861152664180485180487180488 180490180492180495180496180497180498180499190119207648219527 220909

4/3 (1): 185290186024

5/2 (3): 267602681748038214027230979

5/1 (4): 209454

7/3 (4): $\quad 99169 \quad 141224 \quad 182274209430 \quad 209432 \quad 209433 \quad 209434 \quad 209435$ 209439209441209443209445209452209465209466209467209470

7/2 (5): 31010480054800648008480104801148014480154802048021 480264802748029480304803148033480364803748126203386

8/3 (5): $\quad 9671 \quad 30843 \quad 32859 \quad 4801948023 \quad 48139 \quad 104777 \quad 120251 \quad 125664$ $126492129661139227150951167506 \quad 168083196568210028213384226035$ 237892

9/5 (4): 209490

9/4 (5): $33016 \quad 473715271474469101642 \quad 105466 \quad 121614 \quad 128854134821$ $\begin{array}{lllllllllll}150258 & 151436 & 163389 & 172417 & 173187 & 182682 & 186231 & 187691 & 187739 & 196684\end{array}$ 200129201196209429209436209437209438209442209444209446209447 209449209456209457209458209460209463209468209471209472209473 209475209476209479209482209486209487209488209491209495209497 230786232988237349243711245998248481

9/2 (7): 48001175934209455209459209474209481209492209493

10/3 (7): $4 \begin{array}{llllllllll}48002 & 48004 & 48007 & 48009 & 48013 & 48018 & 48025 & 48028 & 48039\end{array}$ 48115180486180489180491180493180494

11/5 (6): $4439799210032 \quad 1810526484349523827748003 \quad 5744564959$ $99193105840106360118403120639140934146096 \quad 150539162959175111$

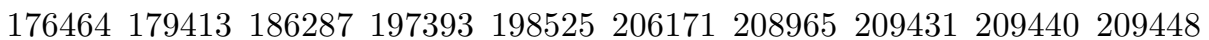
209451209453209461209462209464209477209478209480209483209484 209485209494209498211894213992240485242608247708248231

11/3 (8): 6572098009141845161599 